\title{
The Predator becomes the Prey: Regulating the Ubiquitin System by Ubiquitylation and Degradation
}

\author{
Allan M. Weissman ${ }^{1,{ }^{*}}$, Nitzan Shabek ${ }^{2}$, and Aaron Ciechanover ${ }^{2,{ }^{*}}$ \\ ${ }^{1}$ Laboratory of Protein Dynamics and Signaling, National Cancer Institute, Frederick, Maryland \\ 21702, USA \\ ${ }^{2}$ Cancer and Vascular Biology Research Center, The Rappaport Faculty of Medicine and \\ Research Institute, Technion-Israel Institute of Technology, Haifa 31096, Israel
}

\section{Abstract}

Ubiquitylation (also known as ubiquitination) regulates essentially all intracellular processes in eukaryotes through highly specific, and often tightly spatially and temporally regulated, modification of numerous cellular proteins. Although most often associated with proteasomal degradation, ubiquitylation frequently serves non-proteolytic functions. In light of its central roles in cellular regulation, it has not been surprising to find that many of the components of the ubiquitin system itself are regulated by ubiquitylation. This observation has broad implications for pathophysiology.

\begin{abstract}
A large number of cellular proteins are modified by ubiquitin and UBLs (ubiquitin-like proteins). These modifications generate a recognition element in trans for different downstream effectors that bind to the conjugated ubiquitin or UBL and affect the fate and/or function of the modified protein. The best-studied result of this modification is the targeting of ubiquitin-tagged proteins for proteasomal degradation. The UPS (ubiquitin-proteasome system) is responsible for the regulated degradation of myriad proteins that control numerous central processes, including, for example, the cell cycle and transcription, as well as protein quality control. In light of the large number of UPS substrates, the high specificity
\end{abstract}

*Corresponding authors: A.M.W. Laboratory of Protein Dynamics and Signaling, National Cancer Institute, Frederick, MD 21702, USA., Tel: +1-301-846-7540; Fax: +1-301-846-1666; amw@nih.gov, A.C. The Rappaport Faculty of Medicine and Research Institute, Technion-Israel Institute of Technology, POB 9649, Haifa 31096, Israel. Tel: +972-4-829-5427; Fax: +972-4-852-1193; c_tzachy@netvision.net.il.

\section{AUTHORS' DISCLAIMER}

This publically available version of the manuscript represents the authors' edits to the accepted manuscript and has significant variations from the published version.

\section{About the authors}

Allan M. Weissman obtained his B.S. in biochemistry from Stony Brook University, New York, USA, and his M.D. from Albert Einstein College of Medicine, New York. Following training in internal medicine at Washington University, St. Louis, Missouri, USA, he joined the US National Institutes of Health (NIH), where he now is a Laboratory Chief in the National Cancer Institute Frederick, Maryland, USA. His group within the Laboratory of Protein Dynamics and Signaling focuses on the physiological and pathological roles of the ubiquitin-proteasome system and on understanding structure-function relationships for ubiquitin-conjugating enzymes (E2s) and ubiquitin-protein ligases (E3s).

Nitzan Shabek is a post-doctoral fellow in the laboratory of Aaron Ciechanover in the Faculty of Medicine at the Technion in Haifa, Israel. There, he continues the studies that he initiated when he was a graduate student, also with Aaron Ciechanover, on the mechanisms involved in degradation of ubiquitin by the ubiquitin system.

Aaron Ciechanover was born in Haifa, Israel in 1947. He obtained his M.Sc. in biochemistry and his M.D. from the Hebrew University in Jerusalem, Israel, and his D.Sc. from the Technion in Haifa. As a graduate student with Avram Hershko, and in collaboration with Irwin Rose, they discovered that the covalent attachment of ubiquitin to a target protein signals it for degradation, and deciphered the mechanism of conjugation. His laboratory currently focuses on the mechanisms that underlie the degradation of the ubiquitin system's own components, and on the decoding of the polyubiquitin proteolytic signal. For the discovery of the ubiquitin proteolytic system, he was awarded - along with Avram Hershko and Irwin Rose - the 2004 Nobel Prize in Chemistry. 
of their recognition, and the complex processes regulated, it was not surprising to find that the system comprises $\sim 1,000$ components ( $\sim 5 \%$ of the genome), and that aberrations in its function underlie the mechanism of many diseases, including malignancies, inflammatory disorders and neurodegeneration.

The structure of the UPS is hierarchical. Activated ubiquitin is transferred through a cascade from the one predominant ubiquitin-activating enzyme (E1) to one of several of the 30-40 mammalian ubiquitin carrier proteins (ubiquitin-conjugating enzymes (UBCs); E2s), to the substrate, which is bound specifically to one of hundreds of different ubiquitin-protein ligases (E3s). This process is repeated to generate polyubiquitin chains, which are signals that target conjugated proteins for degradation by the $26 \mathrm{~S}$ proteasome. After proteasome targeting, ubiquitin is recycled by DUBs (deubiquitylating enzymes), some of which also function to oppose the action of E3s (Figure 1).

The UPS is highly regulated at multiple levels. An emerging concept is that the specificityconferring components of the UPS, the E3 ligases, as well as other components of the system, can be regulated by ubiquitylation (known also as ubiquitination, the term coined by the discoverers of this modification as related to proteolysis ${ }^{0}$ ) and degradation, either mediated by themselves (self- or auto-ubiquitylation), or by heterologous ligases. However, there are also clear examples where self-ubiquitylation of E3s serves not as a degradation signal, but rather to alter the function of these proteins. This review focuses on examples of both of these concepts, as well as the regulated degradation of ubiquitin and the proteasome.

\section{DEGRADATION OF UBIQUITIN}

The ability of the UPS to carry out its numerous functions depends on free ubiquitin. Cellular ubiquitin levels are regulated by synthesis and degradation, as well as by rates of protein ubiquitylation and deubiquitylation. Although ubiquitin levels vary among tissues, developmental stage and with differentiation and metabolic activity (for example, under stress, when proteolysis rates increase, free ubiquitin levels fall) ${ }^{1,2}$, the ratio of free to protein-conjugated ubiquitin is approximately 1:1 (REFS 3,4). As ubiquitin is physically stable and remains properly folded and active after exposure to extreme conditions ${ }^{5,6}$, it was thought to be resistant to catabolism. However, ubiquitin has a finite half-life, and its degradation and steady state level reflects overall protein turnover ${ }^{3,7,8}$. Ubiquitin can be also depleted when it is not properly removed and recycled from targeted degraded substrates (REF. 9 and below).

Ubiquitin can be degraded by the $26 \mathrm{~S}$ proteasome in three ways: as a monomer; along with its conjugated substrate through a 'piggyback' mechanism; and as a linear fusion protein with a short C-terminal tail, or as part of an isopeptide conjugate with a short peptide ${ }^{1,10,11}$ (N.S. and A.C. unpublished observations) (Figure 2A).

Degradation of monomeric ubiquitin-Ubiquitin is relatively stable and has a half-life of $\sim 10$ hours $1,3,7,8,10$. Its degradation requires ATP and is mostly proteasome-mediated ${ }^{1,3,8}$. Since monomeric ubiquitin binds to the proteasome but does not have a long enough 'tail' to allow it to be 'pulled' into the $20 \mathrm{~S}$ catalytic particle (CP), it itself has to be ubiquitylated in order to be degraded. This provides the tagged ubiquitin with sufficient freedom of movement to allow for entry into the proteasomal CP. Ubiquitylation is catalyzed by a specific E2, E2-25K (also known as UBE2K), which catalyzes E3-independent 'canonical' proteasome-targeting K48-linked chains on monomeric ubiquitin ${ }^{12}$, and by the HECT (Homologous to the E6-AP C-Terminus) domain E3 TRIP12 (ThyRoid hormone-Interacting Protein 12), which can also ubiquitylate ubiquitin and target it for degradation ${ }^{13}$. The relative importance of these two modes of ubiquitylation of ubiquitin (E3-independent and - 
dependent) to the degradation of monomeric ubiquitin, and the overall contribution of the degradation of monomeric ubiquitin to its catabolism are not known.

Intriguingly, although ubiquitin is ubiquitylated to become a target substrate, it has also been shown that ubiquitin can exist as 'unanchored' free polyubiquitin chains. Although the physiological role(s) of the low steady state levels of these chains remains elusive, several functions have been attributed to them, including involvement in NF- $\mathrm{KB}$ signaling ${ }^{14}$, and serving as a an easily accessible source of monomeric ubiquitin when disassembled by DUBs $^{15}$.

Degradation of ubiquitin with its target substrate-Ubiquitin can be degraded in a piggyback mechanism, along with its target substrate. The accelerated degradation of cellular proteins that occurs with various forms of cellular stress, probably owing to misfolding or other forms of protein damage, is accompanied by accelerated degradation of ubiquitin ${ }^{1,3,8}$. Also, deletion of proteasome-associated DUBs, such as yeast Ubp6 (USP14 in mammals) and UCH37 - which presumably functions with the proteasome subunit RPN11 to remove ubiquitin chains prior to entry of the substrate into the 20S CP - accelerates substrate degradation and results in depletion of ubiquitin ${ }^{9,16-19}$. Interestingly, depletion of ubiquitin results in transcriptional upregulation of Ubp6 (REF. 20), suggesting an autoregulatory feedback loop. Also, Ubp6 acts non-catalytically to slow protein degradation by decreasing flux of proteins through the proteasome, possibly to allow efficient removal and recycling of ubiquitin from the chains tagged to the substrate before its entry into the 20S CP to be degraded ${ }^{21}$. Using a reconstituted cell free system, it has been shown that addition of bona fide substrates of the UPS enhances proteasome-dependent degradation of ubiquitin, when preceded by conjugation of the degraded ubiquitin to the substrates. This finding provides direct evidence in support of the piggyback mechanism ${ }^{10}$. It is assumed that part of the chain, probably the proximal part, which is closer to the substrate, is probably degraded with the substrate, whereas the other moieties, probably the ones that are more distal to the substrate, are 'rescued' and recycled.

An interesting question is whether this piggyback mode of degradation serves a mechanistic role and is a consequence of the need to degrade the substrate, or whether it is a by-product of the proteolytic process. It is possible that part of the ubiquitin chain must always be present on the substrate to secure its binding to the proteasome, and its premature removal can result in detachment of the substrate before complete entry into the proteasome. It has been reported recently that the initial, relatively weak and reversible association of the conjugated substrate with the proteasome, which is mediated by the ubiquitin chain, is followed by a tighter association between substrate and the proteasome that requires ATP hydrolysis and a loosely folded domain on the protein, but appears to be ubiquitinindependent ${ }^{22}$. Thus, according to this study, it is possible that, mechanistically, it is not necessary to degrade part of the ubiquitin chain along with the tagged substrate, and it can be completely rescued and/or detached while the substrate, which is now bound in a tighter association that does not require ubiquitin, is degraded. However, as experimental evidence suggests that part of the chain is degraded along with the substrate ${ }^{10}$, it is possible that the movement of the substrate into the $\mathrm{CP}$ can be more rapid than the hydrolytic activity of proteasomal DUBs, and therefore part of the chain cannot be rescued. Alternatively, the chain is required throughout most of the proteolytic process, cannot be released earlier and is, therefore, partially degraded. Additional detailed mechanistic studies will be necessary in order to dissect the role of the polyubiquitin chains once the substrate has reached the interior of the $\mathrm{CP}$ and degradation has been initiated. Besides DUBs, other proteins have recently been found to be involved in regulating ubiquitin homeostasis. These include RFU1 (Regulator of Free Ubiquitin chains 1), which inhibits the DUB Doa4 and probably controls the equilibrium between monomeric ubiquitin and free ubiquitin chains ${ }^{15}$. 
Degradation of ubiquitin fused or conjugated to short peptides-Several studies have demonstrated that ubiquitin with a C-terminal extension that is longer than 20 amino acids is degraded efficiently by the proteasome, and that the degradation is not dependent on further ubiquitylation ${ }^{1,10,11}$; however, ubiquitin with a shorter extension is stable and not degraded. Similar findings have been shown using ubiquitin synthetically conjugated with an isopeptide bond to short peptides (N.S. and A.C., unpublished observations) ${ }^{23}$. The in vivo sources of these ubiquitin-bound peptides (which were synthetically generated as model substrates in the studies described) are unknown, and they have not been isolated from cells. They could be intermediates of proteasomal degradation of larger cellular substrates in which the ubiquitin moiety is bound to either an internal Lys residue of the substrate via an isopeptide bond, or to its $\mathrm{N}$-terminal residue via a linear peptide bond ${ }^{24}$. Such ubiquitin conjugates can also be products of the ubiquitylation of peptides that might either have their own cellular function or are derived from larger proteins via different processing or destructive processes. Thus, to be removed, these peptides must undergo ubiquitylation to be targeted for proteasomal degradation. Although short peptides to which ubiquitin is bound either linearly or via an isopeptide bond have not been isolated and/or characterized in mammalian cells, evidence for their existence was found in yeast cells lacking Doa4 (REF. 25), which is a DUB implicated in endocytosis and vacuolar degradation ${ }^{26}$.

An important implication of the efficient degradation of C-terminally tailed ubiquitin is that it defines the two minimal requirements for proteasomal recognition and degradation of intact substrates: a proteasomal-binding domain (ubiquitin) and an unstructured tail that is long enough ( $>20$ residues) to allow the molecule to physically extend through the $19 \mathrm{~S}$ regulatory particle (RP) into the $\mathrm{CP}^{27}$ (Figure $2 \mathrm{Ac}$ ). The requirement for an unstructured tail has been demonstrated for several model substrates. Whether this concept holds true in cells for polyubiquitylated and normally folded cellular proteins awaits determination. For such proteins, an important problem is determining the mechanism (or mechanisms) that unfolds a segment to allow entry of the substrate into the CP. It is possible that ubiquitylation destabilizes the protein and results in an unfolded segment. Alternatively, a chaperoneassisted mechanism could be involved. Also, there could be a role for the ATPases in the base of the 19S subunit in this process.

A naturally occurring pathological variant of ubiquitin is $\mathrm{UBB}^{+1}$, which is ubiquitin that has been extended by a 19 residue C-terminal tail. $\mathrm{UBB}^{+1}$ is synthesized due to a dinucleotide deletion during transcription and is implicated in the pathogenesis of an early onset form of Alzheimer's disease and other neurological and conformational disorders ${ }^{28}$. UBB ${ }^{+1}$ cannot be activated by E1 and therefore is not conjugated to other proteins, but it has been shown to inhibit proteasome function ${ }^{10,29}$. Its toxicity is probably due to two mechanisms: it binds to the proteasome but it is resistant to degradation owing to the short 19 residue tail; and it can be ubiquitylated, which creates $\mathrm{UBB}^{+1}$-anchored ubiquitin chains that are resistant to DUBs. The high affinity of the ubiquitylated $\mathrm{UBB}^{+1}$ with its non-degradable ubiquitin chains for the 26 proteasome makes it an efficient inhibitor of the proteasome ${ }^{10,29}$. Experimental extension of the tail of $\mathrm{UBB}^{+1}$ by a single residue renders the protein susceptible to proteasomal degradation without further ubiquitylation ${ }^{10}$. The finding that tailed ubiquitin is degraded efficiently by the proteasome raises the possibility that polyubiquitylation is required to increase the affinity of large substrates to the proteasome to render proteolysis more efficient, but for short substrates, a single moiety or short oligoubiquitin chains may be sufficient when an adequate unstructured domain is also present. 


\section{Regulating UPS Enzymes by Ubiquitylation}

Specificity in ubiquitylation is determined largely by E3s, of which there are over 600 in humans. These function together with subsets of E2s. Given the exquisite regulation of ubiquitylation and its ability to alter both the fate and function of substrates, it is not surprising that E3s might be regulated by targeting themselves for ubiquitylation (regulatory self-ubiquitylation) (Figure 2B). Self-ubiquitylation it is a general property of E3s in vitro ${ }^{30}$ and is often used to confirm that proteins are active ligases, and to assess functionally significant interactions with specific E2s. There are now a number of examples of documented self-ubiquitylation in cells (see Table 1 for examples).

The canonical result of ligase self-ubiquitylation is auto-regulation or targeting for selfdestruction, with the main role of the ligase in most cases being to target heterologous substrates. The complexity of $\mathrm{E} 3$ regulation is further accentuated by increasing examples in which an E3 that may or may not self-ubiquitylate, is subject to ubiquitylation by another E3 (regulation by heterologous E3s; Figure 2B), with self- and heterologous ubiquitylation of the same ligase signaling for either the same or different outcomes. Where different outcomes occur, self-ubiquitylation has been shown to serve regulatory, non-proteolytic functions, whereas the heterologous modification targets for degradation (discussed below; Table 1).

The complex relationship between the multisubunit APC/C (Anaphase Promoting Complex/ Cyclosome) and the SCF (Skp1-Cullin-F-box) families of E3s throughout the cell cycle provides the ultimate example of both self- and heterologous regulation of E3s by ubiquitylation and proteasomal degradation, in this case to ensure regulated cell proliferation and genomic integrity (Box 1). Similarly, DUBs and E2s are also subject to ubiquitylation with evidence, at least in one case, suggesting E3-independent self-ubiquitylation of an E2 (REF. 31) (Table 1 and Box 1). There are also examples where enzymes involved in UBL modification of proteins are targets for ubiquitylation or vice versa. The most extensivelystudied UBL-mediated modification of E3s, and the one with the widest range of known consequences, is the activating modification of the cullin subunits of the CRL (cullin-RING ligase) superfamily of E3s by the UBL Nedd8 (Table 1). Moreover, an increasing number of proteins that include ubiquitin receptor domains (reviewed in REF. 32) are also ubiquitylation substrates.

\section{Box 1}

\section{Cross-regulation of ubiquitin ligase complexes during the cell cycle}

The APC/C (Anaphase Promoting Complex/Cyclosome) is central to cell cycle regulation. The 13 subunit APC/C includes either of two substrate recognition elements with distinct specificities. $\mathrm{APC} / \mathrm{C}^{\mathrm{CDC} 20}$ and $\mathrm{APC} / \mathrm{C}^{\mathrm{CDH} 1}$ are tightly regulated to ensure appropriate cell cycle-dependent degradation of substrates (reviewed in REF. 101). The cullin-containing SCF (Skp1-Cul1-F-box proteins) E3 complexes are also essential for cell cycle progression, with the levels (Skp2) and activities towards specific substrates $(\beta-\mathrm{TrCP})$ of SCF E3s regulated during the cell cycle. APC/C and SCF E3s are crossregulated to ensure correct cell cycle progression (see the figure, APC components - red, SCF components - blue). In late G2 and early M-phase the APC/C is inactivated by the spindle assembly checkpoint (SAC; also known as the mitotic checkpoint complex (MCC)) pending alignment of chromosomes along the mitotic spindle (1). Once this occurs, $\mathrm{APC} / \mathrm{C}^{\mathrm{Cdc} 20}$ is activated and targets substrates essential for completion of mitosis (reviewed in REF.101) (2). After mitosis APC/C ${ }^{\mathrm{Cdc} 20}$ is inactivated (reviewed in REF. 102) through replacement of $\mathrm{Cdc} 20$ by $\mathrm{Cdh} 1$, which, once dephosphorylated, can associate with the APC/C (3). This dephosphorylation is an indirect effect of APC/ 
$\mathrm{C}^{\mathrm{Cdc} 20}$, which targets cyclin A for degradation, thereby inactivating the kinase Cdk1, which is critical for Cdh1 phosphorylation (reviewed in REF.101). APC/C $\mathrm{C}^{\mathrm{Cdh} 1}$ further inactivates $\mathrm{APC} / \mathrm{C}^{\mathrm{Cdc} 20}$ by targeting $\mathrm{Cdc} 20$ for polyubiquitylation (Ubn) and degradation beginning in telophase and continuing in G1 (4). To prevent premature G1 to S progression, the Cyclin E-Cdk2 complex is kept inactive by the G1-S CDK inhibitor p27 ${ }^{\mathrm{KIP}}$. A key E3 for $\mathrm{p} 27^{\mathrm{KIP}}$ is, SCF ${ }^{\mathrm{Skp} 2}$ (REFS 103-105). To maintain $227^{\mathrm{KIP}}$ levels, Skp2 is targeted by APC/C $\mathrm{C}^{\mathrm{Cdh} 1}$ during G1 (REFS 106,107) (5). As G1 ends, APC/C Cdh1 inactivated through multiple mechanisms, including targeting of the E2 UbcH10 (known also as $\mathrm{E} 2 \mathrm{C}$ or $\mathrm{E} 2 \mathrm{X})^{108}$ for degradation by $\mathrm{APC} / \mathrm{C}^{\mathrm{Cdh} 1}(\mathbf{6})$, and ubiquitylation of $\mathrm{Cdh} 1$ by both APC/C ${ }^{C d h 1}$ (REF. 109) and an unidentified SCF E3 ligase ${ }^{110} \mathbf{( 7 , 8 )}$. This allows accumulation of $\mathrm{SCF}^{\mathrm{Skp} 2}$ and $\mathrm{p} 27$ degradation, which facilitates transition into $\mathrm{S}$ phase (9). Synthesis of the APC/C 'pseudosubstrate' EMI1 initiates in late G1. EMI1 binds $\mathrm{APC} / \mathrm{C}^{\mathrm{Cdh} 1}$ during late $\mathrm{G} 1$ and $\mathrm{S}$, contributing to $\mathrm{APC} / \mathrm{C}^{\mathrm{Cdh} 1}$ inactivation (REFS 111-113, reviewed in REF. 114) (10). In some cells, EMI1 similarly inhibits APC/C Cdc20 (11). In early M, EMI1 is phosphorylated by Cdk and PLK1 leading to its targeting for degradation by SCF ${ }^{\beta-T r C P}$ (REFS.115-118) (12). Until prometaphase, the SAC/MCC is the primary means by which $\mathrm{APC} / \mathrm{C}^{\mathrm{Cdc} 20}$ is held in check (1).

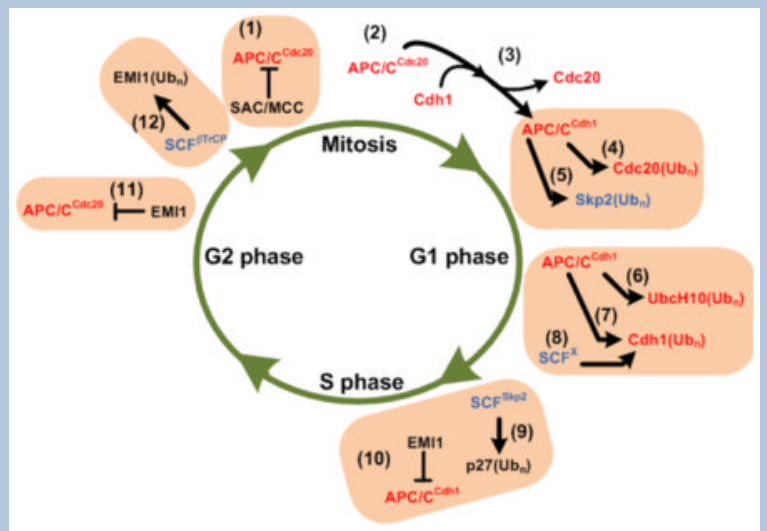

Self-ubiquitylation in regulating the tumor suppressor p53-Regulatory selfubiquitylation (Figure 2B) is illustrated by considering the control of the p53. This protein, known as the guardian of the genome, is normally maintained at low levels (largely due to the UPS) to allow normal cell growth and proliferation; however, under conditions of genotoxic stress its degradation is suppressed through a variety of mechanisms, leading to upregulation of genes that result in either growth arrest or apoptosis. In this regard mutation or loss of p53 expression is a common feature of many malignancies (reviewed in REF.33).

Although a number of E3s have been suggested to be involved in p53 ubiquitylation, Mdm2 (Mouse Double Minutes 2; often referred to as $\mathrm{Hdm} 2$ in humans) is of unquestionable importance. Mdm2 is a RING finger E3 that can form homodimers through its RING finger and binds directly to $\mathrm{p} 53$, which exists primarily as a homotetramer. Mdm2 targets multiple C-terminal lysines of p53 for ubiquitylation (reviewed in REF. 34). Mdm2 undergoes selfubiquitylation in vitro ${ }^{35,36}$ and targets itself and $\mathrm{p} 53$ for RING finger-dependent degradation in vivo ${ }^{35}$. The closely related protein, $\mathrm{MdmX}$ (Mdm4; HdmX/Hdm4 in humans), has little propensity to form homodimers but rather forms heterodimers with Mdm2 through an extended surface centered on their RING fingers ${ }^{37}$. Although the MdmX RING finger lacks ligase activity, the heterodimer is an active $\mathrm{E} 3$ that is generally believed to be the predominant cellular ligase for p53 (Ref. 38, reviewed in REFS 33,34). Mdmx can also directly bind p53 in a fashion similar to Mdm2. Notably, MdmX lacks the nuclear export 
and nuclear and nucleolar localization signals that are characteristic of Mdm2. MdmX can also be ubiquitylated and targeted for degradation by Mdm2, presumably in the context of Mdm2-MdmX heterodimers (reviewed in REFS 33,34). Consistent with this, expression of MdmX decreases ubiquitylation and degradation of Mdm2, presumably by competing with $\mathrm{Mdm} 2$ in the formation of dimers and thereby decreasing the capacity of one Mdm2 molecule to ubiquitylate another. There is evidence that the Mdm2:MdmX ratio plays important roles in p53 ubiquitylation and degradation. MdmX enhances the activity of Mdm2 towards p53 (REFS 39, 40). However, MdmX has also been associated with stabilization of p53 (REF. 41). This role is attributed to the attenuation of nuclear export of p53 by Mdm2, which is believed to lead to degradation of p53 in the cytoplasm. Thus, while it is critical for issues of p53 stability/degradation and localization that Mdm2 and MdmX be maintained in a proper stoichiometric relationship, exactly how the relationship affects p53 function remains an area of intense investigation. An additional level of complexity in the relationship between $\mathrm{p} 53, \mathrm{Mdm} 2$ and MdmX is illustrated by the function of the DUB USP7/HAUSP. This DUB, which was first shown to deubiquitylate p53, has as its primary activity, under non-stressed conditions, the deubiquitylation and stabilization of Mdm2 and $\mathrm{MdmX}^{42,43}$. However, in response to genotoxic stress, phosphorylation of Mdm2 and MdmX leads to decreased HAUSP association and to their destabilization, which further contributes to 53 activation by ATM and downstream kinases ${ }^{44,45}$.

Heterologous and self-ubiquitylation in regulating transmembrane signalingUbiquitylation plays important roles in the regulation of transporters, receptors and associated components of signaling pathways at the plasma membrane and in early endosomes. In general, this modification downregulates these molecules by targeting them for lysosomal/vacuolar degradation (reviewed in REF. 46). Prominent among E3s acting in these pathways are members of the Nedd4 (Neural precursor cell Expressed Developmentally Down-regulated protein 4) family of HECT E3s and the Cbl (Casitas Blineage Lymphoma) family of RING finger E3s.

The Nedd4 family (yeast ortholog is Rsp5) has nine members; prominent among these are the functionally distinct Nedd4-1 and Nedd4-2 (also known as Nedd4L) isoforms and Itch. Nedd4 family members are generally characterized by an N-terminal C2 domain, 2 to $4 \mathrm{WW}$ domains and a $\mathrm{C}$-terminal conserved HECT ubiquitin ligase domain. $\mathrm{C} 2$ domains serve as sites for membrane localization by binding to phospholipids and to proteins. Most known substrate interactions occur through the Trp-based WW domains. Like SH3 domains, these generally recognize Pro-containing 'PY' motifs (reviewed in REF. 47). However, these labels belie the combinatorial complexity between various WW domains and PY motifs ${ }^{48}$.

The Cbl family of E3 ligases was characterized consequent to the discovery of v-Cbl as the product of the transforming gene of the murine Cas NS-1 virus (reviewed in REF. 49). Mutations in Cbl that result in loss of its RING finger-dependent E3 activity, including v$\mathrm{Cbl}$, can function as dominant negatives, resulting in sustained signaling. Such mutations can therefore be oncogenic, as evidenced by the association between such Cbl mutations and myeloid leukemias (reviewed in REF. 50). There are three mammalian Cbl proteins: $\mathrm{Cbl}$ (also known as c-Cbl), Cbl-b and Cbl-c (also known as Cbl-3). Each has an N-terminal tyrosine kinase binding (TKB) domain, a conserved RING finger and a Pro-rich region. Both the TKB domain and the Pro-rich region mediate numerous protein interactions ${ }^{51}$. Cbl proteins are recruited through TKB domains to active signaling complexes, most notably to transmembrane RTKs (Receptor Tyrosine Kinases) and receptors linked to tyrosine kinases. They target these receptors and associated components for ubiquitylation, leading to their lysosomal targeting and degradation. Thus, Cbls serve as potential tumor suppressors by attenuating receptor-mediated mitogenic signaling (reviewed in REF. 49). As part of this process $\mathrm{Cbl}$ proteins undergo self-ubiquitylation, leading to their degradation and thereby 
regulating their own levels (Figure 3A - left). The degradation of $\mathrm{Cbl}$ proteins is dependent on their interactions with RTKs (and vice versa) and on Cbl E3 activity. Degradation of activated receptors, as well as associated signaling molecules and recruited $\mathrm{Cbl}$, is blocked by either lysosome or proteasome inhibitors, suggesting coordinated degradation of the signaling complex ${ }^{52}$. Whether this means of $\mathrm{Cbl}$ degradation occurs in lysosomes, proteasomes or both is unclear.

Another mechanism by which Cbl proteins can be targeted for degradation is by interacting with the Nedd4 family of HECT domain E3s, mediated through interactions between the Pro-containing regions of Cbls and the WW domains of Nedd4 family members. Nedd4-1 and Itch interact with and target all three Cbls for ubiquitylation and degradation (Figure 3A - right). This Cbl ubiquitylation leads to proteasomal degradation and is dependent on an intact HECT domain, but independent of a functional RING finger ${ }^{53}$. Thus, Cbl proteins are bona fide Nedd4 and Itch family substrates. The consequences of this interaction include delayed downregulation of activated EGFR (Epidermal Growth Factor Receptor) and consequently sustained MAP (Mitogen Activated Protein) kinase activation ${ }^{53}$. The relationship between the two classes of E3s is asymmetrical; there is little evidence that Nedd 4 family members are targeted by Cbls. Such asymmetry is a recurrent theme in many of the examples in subsequent sections.

The relationship between these ligase families has been confirmed in helper T cells from Nedd $4^{-/}$embryos. These $\mathrm{T}$ cells have decreased activation, as assessed by IL-2 production and increased Cbl-b levels, which inhibits signaling downstream of the TCR (T Cell Antigen Receptor). In addition, siRNA knockdown of Cbl-b increases IL-2 levels ${ }^{54}$. These observations are in accord with evidence implicating Cbl-b as a negative regulator of TCR signaling (reviewed in REF.49,55).

Some insights into the physical and functional relationship between members of these families come from studies on Itch and Cbl. Itch is a substrate for JNK (Jun N-terminal Kinase $)^{56}$, which is activated by a variety of stimuli, including signaling through the EGFR. Itch phosphorylation results in a conformational change that activates Itch as determined by enhanced self-ubiquitylation ${ }^{56}$. This phosphorylation also facilitates binding of $\mathrm{Cbl}$ and other substrates ${ }^{57}$. This suggests a positive feedback loop in which EGFR signaling can be prolonged by downregulation of $\mathrm{Cbl}$ by activated Itch. A further level of regulation arises from the JNK-dependent binding of WW domains of Itch to the DUB USP9x/FAM (mammalian counterpart of Drosophila Fat-Facets). At a minimum, this interaction decreases ubiquitylation of Itch and therefore increases its availability ${ }^{57,58}$. Whether this DUB diminishes Itch-mediated ubiquitylation of $\mathrm{Cbl}$ or increases Cbl ubiquitylation by increasing active Itch remains unanswered. As multiple WW Nedd4 family members can target different $\mathrm{Cbl}$ proteins, it is now of great interest to determine the means by which specificity in interactions between the multiple members of these families is achieved in vivo. Non-mutually exclusive possibilities include: differential expression of family members, post-translational modifications that regulate interactions and spatial juxtaposition. It will also be important to understand the relative roles of self-ubiquitylation and Nedd4 family-mediated ubiquitylation in the dynamic regulation of Cbl levels and how this relates to the oncogenic potential of $\mathrm{Cbl}$ mutations in different tissues.

\section{Heterologous and self-ubiquitylation at the endoplasmic reticulum-}

Ubiquitylation at the ER (endoplasmic reticulum) leading to proteasomal degradation is a way of disposing of misfolded proteins and unassembled protein subunits, as well as proteins of the secretory pathway that require tight regulation. ERAD (ER-Associated Degradation) represents a critical homeostatic and regulatory set of processes. Failure to adequately degrade proteins results in ER stress, which in turn triggers a complex cellular 
response known as the UPR (unfolded protein response) (reviewed in REF. 59). gp78 is a pro-metastatic mammalian polytopic ERAD E3 ${ }^{60}$ that targets substrates by means that are not limited to direct interactions. Recognition of already ubiquitylated proteins may occur via the ubiquitin binding CUE domain of gp $78^{61}$, or proteins may be targeted through colocalization to microdomains of the ER where the degradation and ER dislocation machinery are concentrated ${ }^{62}$. If the levels of gp78 are too high, critical regulatory proteins such as Insig-1 (insulin-induced gene 1; a regulator of cholesterol metabolism) and KAI1/ CD82 (a metastasis suppressor) may be inappropriately degraded ${ }^{63,64}$. Presumably as a means to avoid such targeting, gp78 self-ubiquitylates, leading to proteasomal degradation in a manner that is dependent on its RING finger, its CUE domain and its unusual binding site for its cognate E2 (Ube2g2/MmUbc7) known as the G2BR (Ube2g2 Binding Region), which allosterically activates gp78 by increasing the affinity of Ube2g2 for gp78 (Figure $3 \mathrm{~B})$. This leads to both enhanced self-ubiquitylation and degradation of substrates 65,66 . Other mammalian and yeast ERAD E3s have also been shown to undergo self-ubiquitylation (Table 1).

In addition to targeting itself for degradation, gp78 is also targeted for ubiquitylation and proteasomal degradation by another mammalian polytopic ERAD E3, Hrd1 (also known as synoviolin (SYVN or SYNO) in mice) ${ }^{67,68}$. This targeting is unidirectional (Figure 3B) and, unlike the self-ubiquitylation of gp78, is independent of the ligase activity of gp78. Importantly, the targeting of gp78 for degradation is not just a function of acute manipulation, as gp78 in MEFs from $S_{y v n^{-1-}}$ mice is stabilized ${ }^{67}$. Interestingly, coimmunoprecipitation of gp78 with Hrd1 is abrogated by mutation of the gp78 CUE domain $^{68}$, which binds ubiquitin ${ }^{65}$. Whether this CUE domain-mediated interaction contributes to gp78 being targeted for degradation by Hrd1, or simply reflects the intermolecular 'glue' provided by domains such as CUE that bind ubiquitin chains remains to be determined. The significance of this targeting is underscored by the finding that the alteration of gp78 levels by Hrd1 impacts gp78 substrates but not those of Hrd1 (REF. 67).

Unlike gp78, endogenous Hrd1 is a relatively stable protein. Studies on its similarly stable yeast counterpart Hrd1p (also known as Der3p) ${ }^{69}$, which has a critical role in targeting 3hydroxyl-3-methylglutaryl (HMG) CoA reductase and thereby regulating sterol synthesis, have led to important insights into the determinants of stability for Hrd1p. In particular, the stability of this yeast RING finger E3 was found to be dependent on its stoichiometric relationship with its polytopic binding partner Hrd3p, with loss of Hrd3p leading to Hrd1p degradation ${ }^{69}$; recently a similar role has been shown for Sel1L, which is a mammalian ortholog of $\mathrm{Hrd} 3 \mathrm{p}^{70}$. The degradation of Hrd1p is dependent on a third transmembrane component of the HRD1 ligase complex USA1 ${ }^{71}$, which is necessary for its normal function in ERAD ${ }^{71,72}$. USA1p serves as part of the scaffold for oligomerization of the HRD1 complex ${ }^{72}$. USA1p and particularly its N-terminal ubiquitin-like domain (UBD) is required for the self-ubiquitylation and proteasomal degradation of $\mathrm{Hrd}_{1} \mathrm{p}^{71}$. Further, consistent with a role in oligomerization, this USA1p-dependent self- ubiquitylation of Hrd1p occurs in trans (i.e. one Hrd1p molecule ubiquitylates another) ${ }^{71}$. Thus, these yeast genetic studies illustrate two important concepts: first that other interacting molecules can play a critical role in self-ubiquitylation of ligases leading to degradation (see also apoptosis section below), and second that self-ubiquitylation can clearly occur between two molecules of the same ligase.

Heterologous and self-ubiquitylation in gene regulation-E3s can both selfubiquitylate and be ubiquitylated by heterologous ligases, and the self-ubiquityation can have functions other than targeting the ligases for proteasomal degradation. One such example is the RING1B component of PRC1 (Polycomb Repressive Complex 1), which self-ubiquitylates to increase its activity. The Polycomb Group (PcG) complex regulates 
repression of transcription during Drosophila development via post-translational modifications of nucleosomal histones. There are two PRCs, PRC1 and PRC2. The human PRC1 contains, among other proteins, two RING finger-containing proteins, RING1B and BMI1 (B lymphoma Mo-MLV Insertion). PRC2 contains EZH2 (Enhancer of Zeste 2), which is a histone methyl transferase. The concerted histone-modifying activities of the two complexes silences gene expression. Initially, PRC2 methylates Lys27 on histone H3 (H3K27), leading to the recruitment of PRC1 (Ref.73). Following binding to methylated H3K27, RING1B catalyzes monoubiquitylation of histone H2A on Lys119. Like MdmX, BMI1 lacks intrinsic E3 activity; however, when it dimerizes with RING1B through their RING fingers, it enhances the ligase activity of RING1B towards histone $\mathrm{H}_{2} \mathrm{~A}^{74}$.

Besides catalyzing ubiquitylation of histone H2A, RING1B mediates its own polyubiquitylation. However, the self-ubiquitylation does not target RING1B for degradation, as mutation in the RING finger that abolishes self-ubiquitylation does not affect its proteasomal degradation ${ }^{74}$. It seems that the self-generated ubiquitin chains do not target RING1B for degradation because, rather than forming canonical homogenous K48 or other proteasome-targeting polyubiquitin chains, RING1B generates atypical, mixed and multiply branched ('forked'; in which one ubiquitin moiety can be modified by several ubiquitins anchored to different Lys residues) K6-, K27-, and K48-linked polyubiquitin chains ${ }^{74}$. These forked chains are probably not degraded by the proteasome ${ }^{75}$. Strikingly, this unique modification stimulates the RING1B histone H2A monoubiquitylating activity, thus providing a novel mechanism for ligase activation through this 'non-canonical' selfubiquitylation. This finding raised the hypothesis that RING1B must be targeted for degradation by a heterologous ligase. E6-associated protein (E6-AP; also known as UBE3A) was identified as one ligase that can target RING1B ${ }^{76}$. It generates K48-linked chains on RING1B, which targets the protein for degradation ${ }^{76}$. The self-ubiquitylation of RING1B and the modification by E6-AP both tag the same Lys residues on RING1B, suggesting a high level of regulation of activation versus degradation of RING1B. Both types of chains are disassembled by USP7, which stabilizes RING1B and resets its activity (the regulation of RING1B by ubiquitylation is depicted in Figure 4A). The elevated levels of RING1B and ubiquitylated H2A observed in Purkinje neurons (and other tissues) from E6-AP deficient mice ${ }^{76}$, could play a role in aberrant transcriptional repression. This may have implications for the pathogenesis of Angelman Syndrome, a neurodevelopmental disorder caused by E6AP deficiency.

Heterologous and self-ubiquitylation in apoptosis-The UPS plays major mechanistic roles in regulating apoptosis (reviewed in REFS 77, 78). Critical regulatory proteins involved in cell death pathways are modified and degraded by the ubiquitin system. These include, for example, the pro-survival protein Mcl-1 and the pro-apoptotic proteins Bak and Bax. Key proteins involved in related pathways are also regulated by the UPS and include, among others, p53, cell cycle regulators, DNA damage response pathways components and transcription factors such as NF- $\mathrm{kB}$. Inhibitors of apoptosis proteins (IAPs), which are critical negative regulators of cell death, are RING finger ubiquitin ligases ${ }^{79}$. Among these are XIAP (X chromosome-linked IAP), cIAP1 (cellular IAP1) and cIAP2, which suppress cell death by inactivating pro-apoptotic regulators such as caspases. As regulation of IAPs has important roles in regulating cell death (and survival), it is not surprising that they are regulated by several mechanisms, including self-ubiquitylation to regulate ligase degradation and activity, as well as regulation by heterologous ligases ${ }^{80-82}$. Thus, following an apoptotic stimulus, the pro-apoptotic protein Reaper - and possibly also Hid (Head Involution Defective) and Grim, which are members of the RHG family of proteins, induces accelerated self-ubiquitylation and rapid degradation of Diap1 (Drosophila IAP1). Reaper binds to Diap1 via a short IAP-Binding Motif (IBM) at its N-terminus, a binding that probably facilitates the transfer of a ubiquitin moiety from the E2 to the ligase 
(REF. 81, reviewed in REF. 83). By contrast, the basal self-ubiquitylation catalyzed by the enzyme in the absence of Reaper appears to generate non-canonical K63-linked chains that do not target the ligase for degradation, but rather decrease its activity towards heterologous substrates, such as the caspase Dronc ${ }^{82}$. This suggests yet another novel role for selfubiquitylation in attenuating ligase activity, which may occur under basal conditions (the regulation of Diap1 is depicted in Figure 4B). Under basal conditions it appears that Diap2 ubiquitylates Diap1, targeting it for degradation ${ }^{82}$.

Besides Diap2, Morgue, which has an F-box domain and an E2-like domain that lacks the active site Cys, also targets Diap1 for degradation, probably acting as an SCF E3 (REF. 84). In mammalian cells, cIAP1 and XIAP are specifically ubiquitylated and degraded following induction of apoptosis in thymocytes by glucocorticoids or etoposide. The IAPs catalyze their own ubiquitylation, which requires their RING domain. The self- ubiquitylation and degradation of IAPs may be a key event in the apoptotic program ${ }^{79}$. Similar to the Drosophila RHG family members, in mammalian cells Smac3, and possibly also second mitochondria-derived activator of caspase (SMAC; also known as DIABLO) and ARTS (Apoptosis-Related protein in the TGF- $\beta$ Signaling pathway), stimulate the autoubiquitylating activity, leading to accelerated degradation of XIAP following apoptotic stimuli $^{85}$. Unlike, however, the RHG family member proteins, the mammalian proteins are mitochondrial, and exit the mitochondria in response to the apoptotic stimulus. Also, unlike Diap1, it is not known whether self-ubiquitylation of mammalian IAPs leads also to the synthesis of non-canonical chains that serve non-proteolytic functions.

Because of the need for tight regulation, IAPs are also controlled by heterologous ubiquitylation. For example, XIAP1 is regulated by cIAP1 (REF. 86)(for the regulation of cIAP and XIAP, see also Figure 4C). The targeting of IAPs by one another in both Drosophila and mammalian cells appears to establish regulatory loops within apoptotic pathways that fine tune survival and death signals. XIAP is also regulated by the ubiquitin ligase SIAH1 (Seven In Absentia Homolog 1), a reaction that also appears to be mediated by ARTS, which binds to both proteins and appears therefore to facilitate efficient ubiquitylation $^{87}$.

\section{THE 26S PROTEASOME}

The highly abundant $\sim 2 \mathrm{MDa} 26 \mathrm{~S}$ proteasome is the proteolytic arm of the UPS. It is made of two sub-complexes, the $19 \mathrm{~S}$ regulatory particle (RP) and the 20S catalytic particle (CP), and in many cases two RPs cap either end of a CP. The $\mathrm{CP}$ is made of two $\beta$ rings that contain the catalytic sites, each is made of seven subunits $\left(\beta_{1-7}\right)$ flanked on both sides by two a-rings, also made of seven subunits each. Thus, the structure of the 20S CP is $a_{1-7} \beta_{1-7} \beta_{1-7} a_{1-7}$. The RP includes a 'base' and a 'lid'. The base is composed of a hexameric ring of ATPases that function to unfold the substrate and open the gate of the interlacing Nterminal segments of the a subunits, thus allowing entry of the unfolded substrate into the catalytic chamber. The lid is involved mostly in specific recognition of the ubiquitin signal (reviewed in REF. 88) (for structure of the 26S complex see Figure 5A). Because of its complex structure, numerous targets, and the need for rapid adaptation to various pathophysiological conditions, this multi-catalytic enzyme complex is stable and not regulated by degradation. Rather, it is primarily regulated by compositional variation.

Some of the integral 20S proteolytic subunits can be replaced in an inducible and tissuespecific manner that alters proteolytic specificities and adapts it to changing needs, most notably immune challenges. In addition to the $19 \mathrm{~S}$ cap, other proteins and complexes, such as PA28, bind to the end of the 20 S cylinder and activate it by opening the 'gate'. Furthermore, proteasome-associated DUBs and E3s can remodel substrate-anchored 
polyubiquitin chains, which may modulate their susceptibility for degradation. Other proteins, such as the chaperone Ecm29, stabilize the association between different subcomplexes of the $26 \mathrm{~S}$ proteasome (reviewed in REF. 88). Consistent with its longevity, the proteasome appears to be degraded by the lysosome, probably through microautophagy ${ }^{89}$.

Recent studies reported the specific ubiquitylation of distinct subunits of the proteasome; however, these modifications appear to serve non-proteolytic functions. Monoubiquitylation of RPN10 regulates the ability of this subunit to bind substrates by sterically inhibiting its UIM (ubiquitin-interacting motif) ${ }^{90}$. Ubiquitylation by the RING finger E3 Not4 is essential to the integrity and function of the $26 \mathrm{~S}$ proteasome, probably by affecting the function of Ecm29, such that, in the absence of the ligase, Ecm29 is ubiquitylated and degraded. A ubiquitylation target(s) of Not4 has not been identified; thus, the underlying mechanism of its action is unknown ${ }^{91}$. It is possible that Not4 targets the Ecm 29 ligase, and in the absence of Not4, the ligase targets Ecm29. Cyclin- dependent kinase-associated protein 1 (Cks1) plays a role in transcriptional activation that is independent from its role in regulating the cell cycle ${ }^{92}$. This requires the Cks1 ubiquitin-binding domain, which allows it to bind to the proteasome via its ubiquitylated subunits. Cks1 can probably bind to other ubiquitylated complexes, thus displaying a broad array of transcription-regulating activities.

Several studies suggest that selective degradation of critical components of the $26 \mathrm{~S}$ proteasome, or induced dissociation of sub-complexes, are involved in the regulation of its activity. Treatment of hippocampal neurons with the neurotransmitter NMDA (N-Methyl-DAspartate) leads to dissociation of the 26S to the 19S cap and the 20S core, and to proteasomal degradation of the 19S (REF. 93). The mechanism that underlies the NMDA effect is not known. It is possible that since there is also a decrease in ubiquitin conjugates following NMDA treatment (see below), the effect on the proteasome is indirect, and proteasome levels decrease when there are fewer substrates to degrade. Interestingly, it was reported that binding of polyubiquitylated substrates to the 19S RP activates proteasomal activity. This probably occurs by inducing conformational changes in the 20S CP that stabilize the gate opening of the a subunits and thereby facilitate channeling of substrates into the 20S and their access to its active sites. Although it has not been shown experimentally, this crosstalk between the 19S RP and 20S CP, and the stabilization of protrusion of the N-termini of the a subunits into the 19S RP, may contribute to the strengthening and stabilization of the association between the two sub-complexes 94,95 . Also, since the proteasome is involved in the endocytosis of glutamate receptor (for which NMDA is a ligand), the effect of NMDA on the proteasome may serve to potentiate the excitatory influence of the transmitter by inhibiting receptor endocytosis and subsequent degradation. It is assumed that the $19 \mathrm{~S}$ dissociates into individual subunits prior to its degradation, although evidence for their ubiquitylation is lacking. Along with the 19S, two of its associated E3s, E6-AP and HUWE1 (HECT, UBA, and WWE domain-containing protein 1), are also degraded in response to $\mathrm{NMDA}^{93}$. It is possible that the destruction of the proteasome-associated ligases suppresses conjugation and degradation, and stabilizes a subset of proteins required for synaptic activity during NMDA excitation.

In another study it was reported that activation of apoptosis results in caspase-mediated cleavage of the proteasomal subunits S6' (Rpt5), S5a (Rpn10), and S2 (Rpn1), resulting in proteasome inactivation ${ }^{96}$. As a result, pro-apoptotic proteins, such as Smac that are targeted by the UPS are stabilized, which is assumed to facilitate the execution of the apoptotic program. Interestingly, in myotubes, caspase-3-mediated cleavage of Rpt 2 and Rpt6 increases proteasomal activity. This appears to be a specific feed-forward mechanism that accelerates proteolysis in muscle during catabolic states ${ }^{97}$. Oxidative stress has been shown to induce disassembly of the proteasome to its sub-complexes ${ }^{98}$. It was suggested that this dissociation protects cells by enabling the released 20S CP to degrade the oxidized proteins 
that are generated under these conditions, which bypasses the need for ubiquitylation, as the $20 \mathrm{~S}$ can degrade proteins in a ubiquitin-independent manner in vitro. It is unclear, however, whether the $20 \mathrm{~S}$ can degrade cellular substrates in vivo, as several strong lines of evidence suggest that even unfolded, oxidized and otherwise damaged proteins are degraded in the cell via a ubiquitin-dependent mechanism. Thus, in one study it was demonstrated that degradation of damaged cellular proteins exposed to heat, cadmium or paraquat, required the E2s Ubc4 and Ubc5, the proteasomal subunit RPN10, and the CDC48-UfD1-NPL4 complex ${ }^{99}$. Also, the absolute requirement for ATP for all types of protein degradation suggests a need for ubiquitylation, which is ATP-dependent, and/or the $26 \mathrm{~S}$ complex, which requires ATP for its assembly and function. By contrast, degradation by the $20 \mathrm{~S} \mathrm{CP}$ is energy-independent. An independent study that supports the notion that the 20S proteasome is inactive in cells was described in yeast, when during the stationary phase, the $26 \mathrm{~S}$ proteasome similarly dissociates ${ }^{100}$. In this case, the released $20 \mathrm{~S}$ is inactive, as the $\mathrm{N}$ termini of the a chains at either end of the $\mathrm{CP}$ remain interlaced, thus the entry gate to the $\mathrm{CP}$ is closed. This study suggests that dissociation is essential to slow proteolysis to maintain viability during nutrient shortage in the stationary phase. Regulation of the $26 \mathrm{~S}$ proteasome by association-dissociation of its sub-complexes and degradation of its different subunits is described in Figure 5B.

\section{PERSPECTIVE}

Ubiquitin, ubiquitin-conjugating enzymes and ubiquitin-protein ligases are not exempt from the powerful regulation of protein fate and function that is conferred by the UPS, and it is likely that individual proteasome components are also targeted. Deubiquitinating enzymes are similarly targeted for ubiquitylation, although here the role of ubiquitylation appears to generally not result in proteasomal degradation.

In considering the degradation of ubiquitin, a number of critical questions remain. Perhaps foremost among these is why is ubiquitin degraded. In many ways, one can view this polypeptide as a reversible modifier that should not be consumed as part of the major process that it facilitates - proteasomal degradation. Yet, it is degraded. Is it simply degraded as bystander or is its degradation integral to the mechanism by which ubiquitylated substrates are fed to the $26 \mathrm{~S}$ proteasome? Beyond this, although we know free cellular ubiquitin levels vary with different conditions, we have little insight into how degrading ubiquitin might play a part in cellular homeostasis or might benefit the organism during development, stress and in other settings.

The regulation of E2 and E3s by ubiquitin is a fascinating area where we are clearly just beginning to scratch the surface. We now understand that the self-ubiquitylation that is stimulated in the test tube is more often a reflection of what can occur in vivo than we may have originally perceived. But what cellular factors determine whether any particular E3 will target itself or another E3 for ubiquitylation? And among the types of ubiquitin modifications that can occur through this process, what determines the type of linkage generated and thus the fate of the protein? Although E3s can clearly target one another among the examples discussed herein, Nedd4s-Cbls, Hrd1-gp78, E6-AP-RING1B, CIAP1XIAP1 and Diap2-Diap1, this apparently occurs in a unidirectional manner. Why this vectorial ubiquitylation occurs is not intuitively obvious as, in many cases, ubiquitylation occurs as a result of juxtaposition of the ligase and any of a number of different lysines or other acceptors on a substrate either naturally or through artificial targeting. One possible explanation is that acceptor residues are somehow exposed on one ligase and not the other when the two associate, although there are certainly a number of other possibilities.

Regardless, of the mechanism of association, the basis for these particular asymmetrical 
relationships between E3s represents new physiological and mechanistic questions to add to the yet unanswered questions about the functions and mechanics of ubiquitylation.

Finally, and related to the previous point, with phosphorylation there exist multiple examples of kinase cascades. In the examples cited herein we deal at the most with two ligases. We should consider the possibility that within the dense cellular milieu, linear or pyramidal cascades of ubiquitylation of E2s and E3s are taking place (Figure 2Bc). These probably include bidirectional ubiquitylation of ligases with the dynamics of ubiquitylation regulated in part by specific DUBs. As with the ubiquitin system in general, should such cascades exist, they are, in all probability, exquisitely regulated in a temporal-spatial manner.

\section{Acknowledgments}

Space constrains do not allow us to cite many of the studies in this evolving, yet already prolific research area, and we apologize for that. Research in the laboratory of A.M.W. is supported by the National Institutes of Health, National Cancer Institute, Center for Cancer Research. Research in the laboratory of A.C. is supported by grants from the Dr. Miriam and Sheldon Adelson Foundation for Medical Research (AMRF), the Israel Science Foundation (ISF), the German-Israeli Foundation for Research and Scientific Development (GIF), the DeutschIsraelische Projektkooperation (DIP), and Rubicon - the European Union (EU) Network of Excellence studying the Role of Ubiquitin and Ubiquitin-like Modifiers in Cellular Regulation. A.C. is an Israel Cancer Research Fund (ICRF) USA Professor.

\section{GLOSSARY}

\section{UNFOLDED PROTEIN RESPONSE (UPR)}

\section{MICROAUTOPHAGY}

\section{CATALYTIC (CORE) PARTICLE}

\section{REGULATORY PARTICLE (RP)}

A cellular response that is triggered by the accumulation of misfolded proteins in the endoplasmic reticulum (ER) and that results in the transcriptional upregulation of ER chaperones and degradative enzymes and a general inhibition of protein synthesis

Formation of vacuoles containing a small portion of the cytosol that is digested by the lysosomal enzymes following the destruction/dissolution of the surrounding membrane. The process occurs under basal metabolic conditions, and unlike stress-induced macroautophagy, the vacuoles are small, and their generation does not involve formation of new membrane and engulfment and digestion of membrane-limited organelles

The $20 \mathrm{~S}$ core particle of the $26 \mathrm{~S}$ proteasome. It is made of four rings two external $a$ rings that are made each of seven distinct subunits (which are identical between the rings), and two adjacent $\beta$ rings, made also of seven distinct subunits (which are also identical between the rings). Three of the seven $\beta$ subunits are proteases with distinct cleaving activities

The 19S complex of the 26S proteasome consists of two sub-complexes - the base and the lid, which are linked together. The base contains the ATPases that are involved in unfolding of the substrate and in opening the entry to the catalytic 20S sub-complex. The lid contains the polyubiquitin chain-recognizing subunits. The RP also includes DUB(s) that recycle ubiquitin 


\section{CANONICAL UBIQUITIN CHAINS HELPER T CELLS}

UBIQUITYLATION

DEUBIQUITYLATING ENZYME
Lys48-based chains that are well-characterized as being proteasome targeting signals

$\mathrm{T}$ cells that functions as an inducer of the effector cells for humoral and cell-mediated immunity. These cells recognize and bind to antigen

Known also (and originally described) as ubiquitination. This is the multi-step process leading to the modificaiton of a protien with single ubiquitin molecules or chains of ubiquitin

Known also as deubiquitinating enzyme or ubiquitin protease. These enzymes play multiple roles including in processing ubiquitin precursors, in dissassembling and trimming ubiquitin chains and in specifically antagonizing the activity of ubiquitin-protein ligase in general or towards specific substrates

\section{References}

0. Wilkinson KD. The discovery of the ubiquitin-dependent proteolysis. Proc Natl Acad Sci USA. 2005; 102:15280-15282. [PubMed: 16230621]

1. Shabek N, Iwai K, Ciechanover A. Ubiquitin is degraded by the ubiquitin system as a monomer and as part of its conjugated target. Biochem Biophys Res Commun. 2007; 363:425-431. [PubMed: 17870054]

2. Hershko A, Eytan E, Ciechanover A, Haas AL. Immunochemical analysis of the turnover of ubiquitin-protein conjugates in intact cells. Relationship to the breakdown of abnormal proteins. $\mathrm{J}$ Biol Chem. 1982; 257:13964-13970. [PubMed: 6292216] The first description of the role of the ubiquitin proteolytic system in the degradation of proteins in nucleated cells. All prior studies describing the roles of the system were carried out using reticulocytes - the terminally differentiating red blood cell.

3. Haas AL, Bright PM. The dynamics of ubiquitin pools within cultured human lung fibroblasts. J Biol Chem. 1987; 262:345-351. [PubMed: 3025210]

4. Patel MB, Majetschak M. Distribution and interrelationship of ubiquitin proteasome pathway component activities and ubiquitin pools in various porcine tissues. Physiol Res. 2007; 56:341-350. [PubMed: 16792465]

5. Ciechanover A, Elias S, Heller H, Ferber S, Hershko A. Characterization of the heat-stable polypeptide of the ATP-dependent proteolytic system from reticulocytes. J Biol Chem. 1980 255:7525-7528. [PubMed: 6249802] The first detailed characterization of ubiquitin.

6. Vijay-Kumar S, Bugg CE, Cook WJ. Structure of ubiquitin refined at 1.8 A resolution. J Mol Biol. 1987; 194:531-544. [PubMed: 3041007]

7. Carlson N, Rechsteiner M. Microinjection of ubiquitin: intracellular distribution and metabolism in HeLa cells maintained under normal physiological conditions. J Cell Biol. 1987; 104:537-546. [PubMed: 3029141]

8. Hiroi Y, Rechsteiner M. Ubiquitin metabolism in HeLa cells starved of amino acids. FEBS Lett. 1992; 307:156-161. [PubMed: 1322828]

9. Leggett DS, et al. Multiple associated proteins regulate proteasome structure and function. Mol Cell. 2002; 10:495-507. [PubMed: 12408819] Describes Ubp6 as a proteasome-associated DUB and its role in controlling cellular ubiquitin levels and proteasomal degradation by balancing the deubiquitinating and proteolytic activities of the protease.

10. Shabek N, Herman-Bachinsky Y, Ciechanover A. Ubiquitin degradation with its substrate, or as a monomer in a ubiquitination-independent mode, provides clues to proteasome regulation. Proc Natl Acad Sci U S A. 2009; 106:11907-11912. [PubMed: 19581590] Description of degradation 
of ubiquitin as a monomer, as a C-terminally extended molecule, and as part of the substrate-anchored polyubiquitin chain.

11. Verhoef LG, et al. Minimal length requirement for proteasomal degradation of ubiquitin-dependent substrates. FASEB J. 2009; 23:123-133. [PubMed: 18796559]

12. Piotrowski J, et al. Inhibition of the $26 \mathrm{~S}$ proteasome by polyubiquitin chains synthesized to have defined lengths. J Biol Chem. 1997; 272:23712-23721. [PubMed: 9295315]

13. Park Y, Yoon SK, Yoon JB. The HECT domain of TRIP12 ubiquitinates substrates of the ubiquitin fusion degradation pathway. J Biol Chem. 2009; 284:1540-1549. [PubMed: 19028681]

14. Xia ZP, et al. Direct activation of protein kinases by unanchored polyubiquitin chains. Nature. 2009; 461:114-119. [PubMed: 19675569]

15. Kimura Y, et al. An inhibitor of a deubiquitinating enzyme regulates ubiquitin homeostasis. Cell. 2009; 137:549-559. [PubMed: 19410548]

16. Anderson C, et al. Loss of Usp14 results in reduced levels of ubiquitin in ataxia mice. J Neurochem. 2005; 95:724-731. [PubMed: 16190881]

17. Hanna J, Leggett DS, Finley D. Ubiquitin depletion as a key mediator of toxicity by translational inhibitors. Mol Cell Biol. 2003; 23:9251-9261. [PubMed: 14645527]

18. Verma R, et al. Role of Rpn11 metalloprotease in deubiquitination and degradation by the $26 \mathrm{~S}$ proteasome. Science. 2002; 298:611-615. [PubMed: 12183636]

19. Lee MJ, Lee BH, Hanna J, King RW, Finley D. Trimming of ubiquitin chains by proteasomeassociated deubiquitinating enzymes. Mol Cell Proteomics. 2011; 10:R110.003871. [PubMed: 20823120]

20. Hanna J, Meides A, Zhang DP, Finley D. A ubiquitin stress response induces altered proteasome composition. Cell. 2007; 129:747-759. [PubMed: 17512408] Demonstrates that 'ubiquitin stress' induces Ubp6, which rescues ubiquitin from target substrates, thus helping to restore ubiquitin homeostasis.

21. Hanna J, et al. Deubiquitinating enzyme Ubp6 functions noncatalytically to delay proteasomal degradation. Cell. 2006; 127:99-111. [PubMed: 17018280]

22. Peth A, Uchiki T, Goldberg AL. ATP-dependent steps in the binding of ubiquitin conjugates to the 26S proteasome that commit to degradation. Mol Cell. 2010; 40 :671-681. [PubMed: 21095592]

23. Kumar KS, Spasser L, Ohayon S, Erlich LA, Brik A. Expeditious chemical synthesis of ubiquitinated peptides employing orthogonal protection and native chemical ligation. Bioconjug Chem. 2011; 22:137-143. [PubMed: 21235224] Describes a novel synthetic method to generate peptides and proteins to which ubiquitin is attached in an isopeptide bond to a lysine residue that can be inserted at any point of choice along the chain.

24. Ciechanover A, Ben-Saadon R. N-terminal ubiquitination: more protein substrates join in. Trends Cell Biol. 2004; 14:103-106. [PubMed: 15055197]

25. Papa FR, Hochstrasser M. The yeast DOA4 gene encodes a deubiquitinating enzyme related to a product of the human tre-2 oncogene. Nature. 1993; 366:313-319. [PubMed: 8247125]

26. Dupre S, Haguenauer-Tsapis R. Deubiquitination step in the endocytic pathway of yeast plasma membrane proteins: crucial role of Doa4p ubiquitin isopeptidase. Mol Cell Biol. 2001; 21:44824494. [PubMed: 11416128]

27. Prakash S, Inobe T, Hatch AJ, Matouschek A. Substrate selection by the proteasome during degradation of protein complexes. Nat Chem Biol. 2009; 5:29-36. [PubMed: 19029916]

Establishes that two elements are critical for the proteasome to recognize and degrade a target substrate - conjugated ubiquitin and an unstructured tail in the substrate that will allow its entry into the catalytic $20 S$ particle.

28. van Leeuwen FW, Hol EM, Fischer DF. Frameshift proteins in Alzheimer's disease and in other conformational disorders: time for the ubiquitin-proteasome system. J Alzheimers Dis. 2006; 9:319-325. [PubMed: 16914870] Description of the naturally occurring C-terminally extended ubiquitin $\mathrm{UBB}+1$ that inhibits the proteasome as it binds to it, but due to its too short tail (19 residues), it cannot be degraded.

29. Lam YA, et al. Inhibition of the ubiquitin-proteasome system in Alzheimer's disease. Proc Natl Acad Sci U S A. 2000; 97:9902-9906. [PubMed: 10944193] 
30. Lorick KL, et al. RING fingers mediate ubiquitin-conjugating enzyme (E2)-dependent ubiquitination. Proc Natl Acad Sci U S A. 1999; 96:11364-11369. [PubMed: 10500182]

Established that RING finger proteins are generally E3s and that they can mediate self- or auto-ubiquitination in vitro.

31. Ravid T, Hochstrasser M. Autoregulation of an E2 enzyme by ubiquitin-chain assembly on its catalytic residue. Nat Cell Biol. 2007; 9:422-427. [PubMed: 17310239]

32. Dikic I, Wakatsuki S, Walters KJ. Ubiquitin-binding domains - from structures to functions. Nat Rev Mol Cell Biol. 2009; 10:659-671. [PubMed: 19773779]

33. Wade M, Wang YV, Wahl GM. The p53 orchestra: Mdm2 and Mdmx set the tone. Trends Cell Biol. 2010; 20:299-309. [PubMed: 20172729]

34. Lee JT, Gu W. The multiple levels of regulation by p53 ubiquitination. Cell Death Differ. 2010; 17:86-92. [PubMed: 19543236]

35. Fang S, Jensen JP, Ludwig RL, Vousden KH, Weissman AM. Mdm2 is a RING finger-dependent ubiquitin protein ligase for itself and p53. J Biol Chem. 2000; 275:8945-8951. [PubMed: 10722742]

36. Honda R, Yasuda H. Activity of MDM2, a ubiquitin ligase, toward p53 or itself is dependent on the RING finger domain of the ligase. Oncogene. 2000; 19:1473-1476. [PubMed: 10723139]

References 35 and 36 established that Mdm2 can target itself for ubiquitination through its RING finger.

37. Linke K, et al. Structure of the MDM2/MDMX RING domain heterodimer reveals dimerization is required for their ubiquitylation in trans. Cell Death Differ. 2008; 15:841-848. [PubMed: 18219319]

38. Tanimura S, et al. MDM2 interacts with MDMX through their RING finger domains. FEBS Lett. 1999; 447:5-9. [PubMed: 10218570]

39. Linares LK, Hengstermann A, Ciechanover A, Muller S, Scheffner M. HdmX stimulates Hdm2mediated ubiquitination and degradation of p53. Proc Natl Acad Sci U S A. 2003; 100:1200912014. [PubMed: 14507994]

40. Okamoto K, Taya Y, Nakagama H. Mdmx enhances p53 ubiquitination by altering the substrate preference of the Mdm2 ubiquitin ligase. FEBS Lett. 2009; 583:2710-2714. [PubMed: 19619542]

41. Stad R, et al. Mdmx stabilizes p53 and Mdm2 via two distinct mechanisms. EMBO Rep. 2001; 2:1029-1034. [PubMed: 11606419]

42. Cummins JM, Vogelstein B. HAUSP is required for p53 destabilization. Cell Cycle. 2004; 3:689692. [PubMed: 15118411]

43. Li M, Brooks CL, Kon N, Gu W. A dynamic role of HAUSP in the p53-Mdm2 pathway. Mol Cell. 2004; 13:879-886. [PubMed: 15053880]

44. Meulmeester E, Pereg Y, Shiloh Y, Jochemsen AG. ATM-mediated phosphorylations inhibit Mdmx/Mdm2 stabilization by HAUSP in favor of p53 activation. Cell Cycle. 2005; 4:1166-1170. [PubMed: 16082221]

45. Meulmeester E, et al. Loss of HAUSP-mediated deubiquitination contributes to DNA damageinduced destabilization of Hdmx and Hdm2. Mol Cell. 2005; 18:565-576. [PubMed: 15916963]

Reference 42-45 establish the deubiquitination of Mdm2 and MdmX by the DUB HAUSP and the significance of regulation of this association in response to genotoxic stress.

46. Acconcia F, Sigismund S, Polo S. Ubiquitin in trafficking: the network at work. Exp Cell Res. 2009; 315:1610-1618. [PubMed: 19007773]

47. Rotin D, Kumar S. Physiological functions of the HECT family of ubiquitin ligases. Nat Rev Mol Cell Biol. 2009; 10:398-409. [PubMed: 19436320]

48. Macias MJ, Wiesner S, Sudol M. WW and SH3 domains, two different scaffolds to recognize proline-rich ligands. FEBS Lett. 2002; 513:30-37. [PubMed: 11911877]

49. Ryan PE, Davies GC, Nau MM, Lipkowitz S. Regulating the regulator: negative regulation of Cbl ubiquitin ligases. Trends Biochem Sci. 2006; 31:79-88. [PubMed: 16406635]

50. Kales SC, Ryan PE, Nau MM, Lipkowitz S. Cbl and human myeloid neoplasms: the Cbl oncogene comes of age. Cancer Res. 2010; 70:4789-4794. [PubMed: 20501843]

51. Davies GC, et al. Cbl-b interacts with ubiquitinated proteins; differential functions of the UBA domains of c-Cbl and Cbl-b. Oncogene. 2004; 23:7104-7115. [PubMed: 15273720] 
52. Ettenberg SA, et al. Cbl-b-dependent coordinated degradation of the epidermal growth factor receptor signaling complex. J Biol Chem. 2001; 276:27677-27684. [PubMed: 11375397]

53. Magnifico A, et al. WW domain HECT E3s target Cbl RING finger E3s for proteasomal degradation. J Biol Chem. 2003; 278:43169-43177. [PubMed: 12907674] References 52 and 53 respectively establish the RTK-mediated down regulation of $\mathrm{Cbl}$ proteins by selfubiquitination and their ubiqutiination by Nedd4 family members. Reference 53 is the first clear example of targeting of one $\mathrm{E} 3$ family by another.

54. Yang B, et al. Nedd4 augments the adaptive immune response by promoting ubiquitin-mediated degradation of Cbl-b in activated T cells. Nat Immunol. 2008; 9:1356-1363. [PubMed: 18931680]

55. Gay DL, Ramon H, Oliver PM. Cbl- and Nedd4-family ubiquitin ligases: balancing tolerance and immunity. Immunol Res. 2008; 42:51-64. [PubMed: 18827983]

56. Gallagher E, Gao M, Liu YC, Karin M. Activation of the E3 ubiquitin ligase Itch through a phosphorylation-induced conformational change. Proc Natl Acad Sci U S A. 2006; 103:17171722. [PubMed: 16446428]

57. Azakir BA, Angers A. Reciprocal regulation of the ubiquitin ligase Itch and the epidermal growth factor receptor signaling. Cell Signal. 2009; 21:1326-1336. [PubMed: 19341794]

58. Mouchantaf R, et al. The ubiquitin ligase itch is auto-ubiquitylated in vivo and in vitro but is protected from degradation by interacting with the deubiquitylating enzyme FAM/USP9X. J Biol Chem. 2006; 281:38738-38747. [PubMed: 17038327]

59. Tsai YC, Weissman AM. The Unfolded Protein Response, Degradation from Endoplasmic Reticulum and Cancer. Genes Cancer. 2010; 1:764-778. [PubMed: 21331300]

60. Fang S, et al. The tumor autocrine motility factor receptor, gp78, is a ubiquitin protein ligase implicated in degradation from the endoplasmic reticulum. Proc Natl Acad Sci U S A. 2001; 98:14422-14427. [PubMed: 11724934]

61. Morito D, et al. Gp78 cooperates with RMA1 in endoplasmic reticulum-associated degradation of CFTRDeltaF508. Mol Biol Cell. 2008; 19:1328-1336. [PubMed: 18216283]

62. Ye Y, et al. Recruitment of the p97 ATPase and ubiquitin ligases to the site of retrotranslocation at the endoplasmic reticulum membrane. Proc Natl Acad Sci U S A. 2005; 102:14132-14138. [PubMed: 16186510]

63. Lee JN, Song B, DeBose-Boyd RA, Ye J. Sterol-regulated degradation of Insig-1 mediated by the membrane-bound ubiquitin ligase gp78. J Biol Chem. 2006; 281:39308-39315. [PubMed: 17043353]

64. Tsai YC, et al. The ubiquitin ligase gp78 promotes sarcoma metastasis by targeting KAI1 for degradation. Nat Med. 2007; 13:1504-1509. [PubMed: 18037895]

65. Chen B, et al. The activity of a human endoplasmic reticulum-associated degradation E3, gp78, requires its Cue domain, RING finger, and an E2-binding site. Proc Natl Acad Sci U S A. 2006; 103:341-346. [PubMed: 16407162]

66. Das R, et al. Allosteric activation of E2-RING finger-mediated ubiquitylation by a structurally defined specific E2-binding region of gp78. Mol Cell. 2009; 34:674-685. [PubMed: 19560420]

67. Shmueli A, Tsai YC, Yang M, Braun MA, Weissman AM. Targeting of gp78 for ubiquitinmediated proteasomal degradation by Hrd1: cross-talk between E3s in the endoplasmic reticulum. Biochem Biophys Res Commun. 2009; 390:758-762. [PubMed: 19835843]

68. Ballar P, Ors AU, Yang H, Fang S. Differential regulation of CFTRDeltaF508 degradation by ubiquitin ligases gp78 and Hrd1. Int J Biochem Cell Biol. 2010; 42:167-173. [PubMed: 19828134] Reference 67 and 68 establish the regulation of the pro-metastatic ERAD E3, gp78 by HsHrd1/Synoviolin.

69. Gardner RG, et al. Endoplasmic reticulum degradation requires lumen to cytosol signaling. Transmembrane control of Hrd1p by Hrd3p. J Cell Biol. 2000; 151:69-82. [PubMed: 11018054]

70. Iida Y, et al. SEL1L protein critically determines the stability of the HRD1-SEL1L endoplasmic reticulum-associated degradation (ERAD) complex to optimize the degradation kinetics of ERAD substrates. J Biol Chem. 2011; 286:16929-16939. [PubMed: 21454652]

71. Carroll SM, Hampton RY. Usa1p is required for optimal function and regulation of the Hrd1p endoplasmic reticulum-associated degradation ubiquitin ligase. J Biol Chem. 2010; 285:51465156. [PubMed: 19940128] Establishes that the critical yeast ERAD E3 Hrd1p undergoes self- 
ubiquitination in trans in a manner that regulated by a relative lack of Hrd3p and the presence of Usa1p, both of which are components of the HRD1 ubiquitin ligase complex.

72. Horn SC, et al. Usa1 functions as a scaffold of the HRD-ubiquitin ligase. Mol Cell. 2009; 36 :782793. [PubMed: 20005842]

73. Cao R, et al. Role of histone H3 lysine 27 methylation in Polycomb-group silencing. Science. 2002; 298:1039-1043. [PubMed: 12351676]

74. Ben-Saadon R, Zaaroor D, Ziv T, Ciechanover A. The polycomb protein Ring1B generates self atypical mixed ubiquitin chains required for its in vitro histone $\mathrm{H} 2 \mathrm{~A}$ ligase activity. Mol Cell. 2006; 24:701-711. [PubMed: 17157253] Establishes that Ring1B undergoes self-ubiquitination with formation of multiply-branched chains that do not target for degradation but rather activate the ligase.

75. Kim HT, Kim KP, Uchiki T, Gygi SP, Goldberg AL. S5a promotes protein degradation by blocking synthesis of nondegradable forked ubiquitin chains. EMBO J. 2009; 28:1867-1877. [PubMed: 19387488]

76. Zaaroor-Regev D, et al. Regulation of the polycomb protein Ring1B by self- ubiquitination or by E6-AP may have implications to the pathogenesis of Angelman syndrome. Proc Natl Acad Sci U S A. 2010; 107:6788-6793. [PubMed: 20351251] Demonstrates that the stability of Ring1B is regulated by heterologous ligases including the HECT domain E3, E6-AP.

77. Bernassola F, Ciechanover A, Melino G. The ubiquitin proteasome system and its involvement in cell death pathways. Cell Death Differ. 2010; 17:1-3. [PubMed: 20010850]

78. Vucic D, Dixit VM, Wertz IE. Ubiquitylation in apoptosis: a post-translational modification at the edge of life and death. Nat Rev Mol Cell Biol. 2011; 12:439-452. [PubMed: 21697901]

79. Yang Y, Fang S, Jensen JP, Weissman AM, Ashwell JD. Ubiquitin protein ligase activity of IAPs and their degradation in proteasomes in response to apoptotic stimuli. Science. 2000; 288:874877. [PubMed: 10797013] Demonstrates that activation of IAPs by steroids leads to autoubiquitination and induction of apoptosis. Provides a mechanistic description of the deleterious effect of steroids on lymphocytes.

80. Ditzel M, et al. Degradation of DIAP1 by the N-end rule pathway is essential for regulating apoptosis. Nat Cell Biol. 2003; 5:467-473. [PubMed: 12692559]

81. Ryoo HD, Bergmann A, Gonen H, Ciechanover A, Steller H. Regulation of Drosophila IAP1 degradation and apoptosis by reaper and ubcD1. Nat Cell Biol. 2002; 4:432-438. [PubMed: 12021769] Establishes that one mechanism of induction of apoptosis by the small protein Reaper is via its ability to bind and induce self-ubiquitination and subsequent degradation of Diap1.

82. Herman-Bachinsky Y, Ryoo HD, Ciechanover A, Gonen H. Regulation of the Drosophila ubiquitin ligase DIAP1 is mediated via several distinct ubiquitin system pathways. Cell Death Differ. 2007; 14:861-871. [PubMed: 17205079]

83. Steller H. Regulation of apoptosis in Drosophila. Cell Death Differ. 2008; 15:1132-1138. [PubMed: 18437164]

84. Wing JP, et al. Drosophila Morgue is an F box/ubiquitin conjugase domain protein important for grim-reaper mediated apoptosis. Nat Cell Biol. 2002; 4:451-456. [PubMed: 12021772]

85. Fu J, Jin Y, Arend LJ. Smac3, a novel Smac/DIABLO splicing variant, attenuates the stability and apoptosis-inhibiting activity of X-linked inhibitor of apoptosis protein. J Biol Chem. 2003; 278:52660-52672. [PubMed: 14523016]

86. Silke J, Kratina T, Ekert PG, Pakusch M, Vaux DL. Unlike Diablo/smac, Grim promotes global ubiquitination and specific degradation of $\mathrm{X}$ chromosome-linked inhibitor of apoptosis (XIAP) and neither cause apoptosis. J Biol Chem. 2004; 279:4313-4321. [PubMed: 14570909]

87. Garrison JB, et al. ARTS and Siah collaborate in a pathway for XIAP degradation. Mol Cell. 2011; 41:107-116. [PubMed: 21185211]

88. Finley D. Recognition and processing of ubiquitin-protein conjugates by the proteasome. Annu Rev Biochem. 2009; 78:477-513. [PubMed: 19489727]

89. Cuervo AM, Palmer A, Rivett AJ, Knecht E. Degradation of proteasomes by lysosomes in rat liver. Eur J Biochem. 1995; 227:792-800. [PubMed: 7867640] 
90. Isasa M, et al. Monoubiquitination of RPN10 regulates substrate recruitment to the proteasome. Mol Cell. 2010; 38:733-745. [PubMed: 20542005]

91. Panasenko OO, Collart MA. Not4 E3 Ligase Contributes to Proteasome Assembly and Functional Integrity in Part through Ecm29. Mol Cell Biol. 2011; 31:1610-1623. [PubMed: 21321079]

92. Holic R, et al. Cks1 activates transcription by binding to the ubiquitylated proteasome. Mol Cell Biol. 2010; 30:3894-3901. [PubMed: 20516216]

93. Tai HC, Besche H, Goldberg AL, Schuman EM. Characterization of the Brain 26S Proteasome and its Interacting Proteins. Front Mol Neurosci. 2010; 3A detailed analysis of the brain proteasome and its regulation by different stimuli such as oxidative stress and NMDA receptor activity.

94. Peth A, Besche HC, Goldberg AL. Ubiquitinated proteins activate the proteasome by binding to Usp14/Ubp6, which causes 20S gate opening. Mol Cell. 2009; 36 :794-804. [PubMed: 20005843]

95 . Bech-Otschir D, et al. Polyubiquitin substrates allosterically activate their own degradation by the 26S proteasome. Nat Struct Mol Biol. 2009; 16:219-225. [PubMed: 19169257]

96. Sun XM, et al. Caspase activation inhibits proteasome function during apoptosis. Mol Cell. 2004; 14:81-93. [PubMed: 15068805] Describes the regulation of the proteasome during apoptosis.

97. Wang XH, et al. Caspase- 3 cleaves specific $19 \mathrm{~S}$ proteasome subunits in skeletal muscle stimulating proteasome activity. J Biol Chem. 2010; 285:21249-21257. [PubMed: 20424172]

98. Wang X, Yen J, Kaiser P, Huang L. Regulation of the 26S proteasome complex during oxidative stress. Sci Signal. 2010; 3:ra88. [PubMed: 21139140]

99. Medicherla B, Goldberg AL. Heat shock and oxygen radicals stimulate ubiquitin-dependent degradation mainly of newly synthesized proteins. J Cell Biol. 2008; 182:663-673. [PubMed: 18725537]

100. Bajorek M, Finley D, Glickman MH. Proteasome disassembly and downregulation is correlated with viability during stationary phase. Curr Biol. 2003; 13:1140-1144. [PubMed: 12842014] Describes the regulation of the proteasome by starvation.

101. Peters JM. The anaphase promoting complex/cyclosome: a machine designed to destroy. Nat Rev Mol Cell Biol. 2006; 7:644-656. [PubMed: 16896351]

102. Manchado E, Eguren M, Malumbres M. The anaphase-promoting complex/cyclosome (APC/C): cell-cycle-dependent and -independent functions. Biochem Soc Trans. 2010; 38:65-71. [PubMed: 20074037]

103. Carrano AC, Eytan E, Hershko A, Pagano M. SKP2 is required for ubiquitin-mediated degradation of the CDK inhibitor p27. Nat Cell Biol. 1999; 1:193-199. [PubMed: 10559916]

104. Sutterluty H, et al. p45SKP2 promotes $\mathrm{p} 27 \mathrm{Kip} 1$ degradation and induces $\mathrm{S}$ phase in quiescent cells. Nat Cell Biol. 1999; 1:207-214. [PubMed: 10559918]

105. Tsvetkov LM, Yeh KH, Lee SJ, Sun H, Zhang H. p27(Kip1) ubiquitination and degradation is regulated by the SCF(Skp2) complex through phosphorylated Thr187 in p27. Curr Biol. 1999; 9:661-664. [PubMed: 10375532]

106. Bashir T, Dorrello NV, Amador V, Guardavaccaro D, Pagano M. Control of the SCF(Skp2-Cks1) ubiquitin ligase by the APC/C(Cdh1) ubiquitin ligase. Nature. 2004; 428:190-193. [PubMed: 15014502]

107. Wei W, et al. Degradation of the SCF component Skp2 in cell-cycle phase G1 by the anaphasepromoting complex. Nature. 2004; 428:194-198. [PubMed: 15014503] References 106 and 107 establish that $\mathrm{SCF}^{\mathrm{Skp} 2}$ is targeted for degradation by the APC/C.

108. Yamanaka A, et al. Cell cycle-dependent expression of mammalian E2-C regulated by the anaphase-promoting complex/cyclosome. Mol Biol Cell. 2000; 11:2821-2831. [PubMed: 10930472] Provides an example of cell cycle-dependent degradation of an E2 as a means to inactive its cognate $\mathbf{E 3}$.

109. Listovsky T, et al. Mammalian Cdh1/Fzr mediates its own degradation. EMBO J. 2004; 23:16191626. [PubMed: 15029244] Establishes a role the Cdh1 component of the APC/C in its own cell cycle-dependent degradation.

110. Benmaamar R, Pagano M. Involvement of the SCF complex in the control of Cdh1 degradation in S-phase. Cell Cycle. 2005; 4:1230-1232. [PubMed: 16123585] 
111. Hsu JY, Reimann JD, Sorensen CS, Lukas J, Jackson PK. E2F- dependent accumulation of hEmi1 regulates $S$ phase entry by inhibiting APC(Cdh1). Nat Cell Biol. 2002; 4:358-366. [PubMed: 11988738]

112. Reimann JD, Gardner BE, Margottin-Goguet F, Jackson PK. Emi1 regulates the anaphasepromoting complex by a different mechanism than Mad2 proteins. Genes Dev. 2001; 15:32783285. [PubMed: 11751633]

113. Reimann JD, et al. Emi1 is a mitotic regulator that interacts with Cdc20 and inhibits the anaphase promoting complex. Cell. 2001; 105:645-655. [PubMed: 11389834]

114. Di Fiore B, Pines J. Defining the role of Emi1 in the DNA replication-segregation cycle. Chromosoma. 2008; 117:333-338. [PubMed: 18317792]

115. Guardavaccaro D, et al. Control of meiotic and mitotic progression by the $\mathrm{F}$ box protein betaTrcp1 in vivo. Dev Cell. 2003; 4:799-812. [PubMed: 12791266]

116. Margottin-Goguet F, et al. Prophase destruction of Emi1 by the SCF(betaTrCP/Slimb) ubiquitin ligase activates the anaphase promoting complex to allow progression beyond prometaphase. Dev Cell. 2003; 4:813-826. [PubMed: 12791267] References 115 and 116 establish that the APC/C pseudosubstrate and inhibitor, EMI1 is targeted for degradation by SCF ${ }^{\beta-T r C P}$.

117. Moshe Y, Boulaire J, Pagano M, Hershko A. Role of Polo-like kinase in the degradation of early mitotic inhibitor 1, a regulator of the anaphase promoting complex/cyclosome. Proc Natl Acad Sci U S A. 2004; 101:7937-7942. [PubMed: 15148369]

118. Hansen DV, Loktev AV, Ban KH, Jackson PK. Plk1 regulates activation of the anaphase promoting complex by phosphorylating and triggering SCFbetaTrCP-dependent destruction of the APC Inhibitor Emi1. Mol Biol Cell. 2004; 15:5623-5634. [PubMed: 15469984]

119. Xu P, et al. Quantitative proteomics reveals the function of unconventional ubiquitin chains in proteasomal degradation. Cell. 2009; 137:133-145. [PubMed: 19345192]

120. Matsumoto ML, et al. K11-linked polyubiquitination in cell cycle control revealed by a K11 linkage-specific antibody. Mol Cell. 2010; 39:477-484. [PubMed: 20655260]

121. Saeki Y, et al. Lysine 63-linked polyubiquitin chain may serve as a targeting signal for the $26 \mathrm{~S}$ proteasome. EMBO J. 2009; 28:359-371. [PubMed: 19153599]

122. Carvalho AF, et al. Ubiquitination of mammalian Pex $5 p$, the peroxisomal import receptor. J Biol Chem. 2007; 282:31267-31272. [PubMed: 17726030]

123. Cadwell K, Coscoy L. Ubiquitination on nonlysine residues by a viral E3 ubiquitin ligase. Science. 2005; 309:127-130. [PubMed: 15994556]

124. Ishikura S, Weissman AM, Bonifacino JS. Serine residues in the cytosolic tail of the T-cell antigen receptor alpha-chain mediate ubiquitination and endoplasmic reticulum-associated degradation of the unassembled protein. J Biol Chem. 2010; 285:23916-23924. [PubMed: 20519503]

125. Wang X, et al. Ubiquitination of serine, threonine, or lysine residues on the cytoplasmic tail can induce ERAD of MHC-I by viral E3 ligase mK3. J Cell Biol. 2007; 177:613-624. [PubMed: 17502423]

126. Williams C, van den Berg M, Sprenger RR, Distel B. A conserved cysteine is essential for Pex4pdependent ubiquitination of the peroxisomal import receptor Pex5p. J Biol Chem. 2007; 282:22534-22543. [PubMed: 17550898]

127. Tait SW, et al. Apoptosis induction by Bid requires unconventional ubiquitination and degradation of its N-terminal fragment. J Cell Biol. 2007; 179:1453-1466. [PubMed: 18166654]

128. Shimizu Y, Okuda-Shimizu Y, Hendershot LM. Ubiquitylation of an ERAD substrate occurs on multiple types of amino acids. Mol Cell. 2010; 40:917-926. [PubMed: 21172657]

129. Scheffner M, Huibregtse JM, Vierstra RD, Howley PM. The HPV-16 E6 and E6-AP complex functions as a ubiquitin-protein ligase in the ubiquitination of p53. Cell. 1993; 75:495-505. [PubMed: 8221889]

130. Nuber U, Schwarz SE, Scheffner M. The ubiquitin-protein ligase E6-associated protein (E6-AP) serves as its own substrate. Eur J Biochem. 1998; 254:643-649. [PubMed: 9688277]

131. Hassink G, et al. TEB4 is a C4HC3 RING finger-containing ubiquitin ligase of the endoplasmic reticulum. Biochem J. 2005; 388:647-655. [PubMed: 15673284] 
132. Wang L, et al. Degradation of the bile salt export pump at endoplasmic reticulum in progressive familial intrahepatic cholestasis type II. Hepatology. 2008; 48:1558-1569. [PubMed: 18798335]

133. Zavacki AM, et al. The E3 ubiquitin ligase TEB4 mediates degradation of type 2 iodothyronine deiodinase. Mol Cell Biol. 2009; 29:5339-5347. [PubMed: 19651899]

134. Zhou P, Howley PM. Ubiquitination and degradation of the substrate recognition subunits of SCF ubiquitin-protein ligases. Mol Cell. 1998; 2:571-580. [PubMed: 9844630]

135. Li X, Yang Y, Ashwell JD. TNF-RII and c-IAP1 mediate ubiquitination and degradation of TRAF2. Nature. 2002; 416:345-347. [PubMed: 11907583]

136. Wu W, et al. HERC2 is an E3 ligase that targets BRCA1 for degradation. Cancer Res. 2010; 70:6384-6392. [PubMed: 20631078]

137. Kee Y, Kim JM, D'Andrea AD. Regulated degradation of FANCM in the Fanconi anemia pathway during mitosis. Genes Dev. 2009; 23:555-560. [PubMed: 19270156] Establishes that a critical component of the Fanconi Anemia ubiquitin ligase is targeted for degradation by $\mathrm{SCF}^{\beta-T r C P}$ as a way of inactivating the FANC E3 during mitosis and preventing chromosomal abnormalities.

138. Lilley CE, et al. A viral E3 ligase targets RNF8 and RNF168 to control histone ubiquitination and DNA damage responses. EMBO J. 2010; 29:943-955. [PubMed: 20075863] This provides an example of how a virally-encoded E3 targets critical RING finger E3s involved in the DNA damage response for degradation.

139. Nathan JA, et al. The ubiquitin E3 ligase MARCH7 is differentially regulated by the deubiquitylating enzymes USP7 and USP9X. Traffic. 2008; 9:1130-1145. [PubMed: 18410486]

140. Wada K, Kamitani T. Autoantigen Ro52 is an E3 ubiquitin ligase. Biochem Biophys Res Commun. 2006; 339:415-421. [PubMed: 16297862]

141. Wada K, Niida M, Tanaka M, Kamitani T. Ro52-mediated monoubiquitination of IKK \{beta\} down-regulates NF-\{kappa\}B signalling. J Biochem. 2009; 146:821-832. [PubMed: 19675099]

142. Shen $\mathrm{C}$, et al. Calcium/calmodulin regulates ubiquitination of the ubiquitin-specific protease TRE17/USP6. J Biol Chem. 2005; 280:35967-35973. [PubMed: 16127172]

143. Meray RK, Lansbury PTJ. Reversible monoubiquitination regulates the Parkinson diseaseassociated ubiquitin hydrolase UCH-L1. J Biol Chem. 2007; 282:10567-10575. [PubMed: 17259170]

144. Todi SV, et al. Ubiquitination directly enhances activity of the deubiquitinating enzyme ataxin-3. EMBO J. 2009; 28:372-382. [PubMed: 19153604]

145. Todi SV, et al. Activity and cellular functions of the deubiquitinating enzyme and polyglutamine disease protein ataxin-3 are regulated by ubiquitination at lysine 117. J Biol Chem. 2010; 285:39303-39313. [PubMed: 20943656]

146. Ying Z, et al. Gp78, an ER associated E3, promotes SOD1 and ataxin-3 degradation. Hum Mol Genet. 2009; 18:4268-4281. [PubMed: 19661182]

147. Wada K, Kamitani T. UnpEL/Usp4 is ubiquitinated by Ro52 and deubiquitinated by itself. Biochem Biophys Res Commun. 2006; 342:253-258. [PubMed: 16472766]

148. Boutell C, Canning M, Orr A, Everett RD. Reciprocal activities between herpes simplex virus type 1 regulatory protein ICP0, a ubiquitin E3 ligase, and ubiquitin-specific protease USP7. J Virol. 2005; 79:12342-12354. [PubMed: 16160161]

149. Lee HJ, Kim MS, Kim YK, Oh YK, Baek KH. HAUSP, a deubiquitinating enzyme for p53, is polyubiquitinated, polyneddylated, and dimerized. FEBS Lett. 2005; 579:4867-4872. [PubMed: 16111684]

150. Denuc A, Bosch-Comas A, Gonzalez-Duarte R, Marfany G. The UBA-UIM domains of the USP25 regulate the enzyme ubiquitination state and modulate substrate recognition. PLoS ONE. 2009; 4:e5571. [PubMed: 19440361]

151. Bazirgan OA, Hampton RY. Cue1p is an activator of Ubc7p E2 activity in vitro and in vivo. J Biol Chem. 2008; 283:12797-12810. [PubMed: 18321851]

152. Kostova Z, Mariano J, Scholz S, Koenig C, Weissman AM. A Ubc7p-binding domain in Cue1p activates ER-associated protein degradation. J Cell Sci. 2009; 122:1374-1381. [PubMed: 19366730] 
153. Kreft SG, Hochstrasser M. An unusual transmembrane helix in the Doa10 ERAD ubiquitin ligase modulates degradation of its cognate E2. J Biol Chem. 2011

154. Ho CW, Chen HT, Hwang J. UBC9 autosumoylation negatively regulates sumoylation of septins in Saccharomyces Cerevisiae. J Biol Chem. 2011

155. Pichler A, et al. SUMO modification of the ubiquitin-conjugating enzyme E2-25K. Nat Struct Mol Biol. 2005; 12:264-269. [PubMed: 15723079]

156. Duda DM, et al. Structural regulation of cullin-RING ubiquitin ligase complexes. Curr Opin Struct Biol. 2011; 21:257-264. [PubMed: 21288713] 


\section{Online 'at-a-glance' summary}

- Monomeric ubiquitin is relatively stable, however it appears to be degraded by the proteasome following its own ubiquitylation mediated by the TRIP12 ligase. Ubiquitin is also degraded via two other mechanisms: along with the target substrate as part of the polyubiquitin chain attached to it, and along with a peptide attached either linearly or in an isopeptide bond to its C-terminal Gly residue.

- Ubiquitin-protein ligases (E3s) are largely responsible for conferring substrate specificity to the ubiquitin-proteasome system (UPS). An increasing number of these are being shown to be subject to selfubiquitylation (autoubiquitylation), ubiquitylated by heterologous ligases or both. In some cases both selfubiquitylation and ubiquitylation by heterologous ligases lead to degradation of the protein. In other cases self-ubiquitylation can regulate the cellular function of the ligase while ubiquitylation by a heterologous E3 results in degradation of the target ligase.

- Other components of the ubiquitin proteasome system including ubiquitincarrier enzymes (E2s) and deubiquitylating enzymes are also subject to ubiquitylation.

- Components of the ubiquitin system are also subject to modification by other ubiquitin-like protein modifiers.

- The 26S proteasome is a stable, long-lived complex, and is probably degraded via microautophagy. As part of the response to some specific cellular signals, such as oxidative stress, starvation, and stimulation of the N-methyl-D-aspartate (NMDA) receptor, it is disassembled into its two subcomplexes, the 19S regulatory particle (RP) and the $20 \mathrm{~S}$ catalytic (or core) particle (CP). The RP is probably dissembled into its individual subunits, which are degraded by the proteasome following ubiquitylation. Caspase-mediated cleavage of specific 19S subunits has also been shown to regulate proteasomal activity under certain conditions.

- The effect of disassembly of the $26 \mathrm{~S}$ proteasome on the $20 \mathrm{~S}$ complex has remained unclear: in some cases it was shown to inhibit its activity, to avoid damage of uncontrolled degradation, whereas in others it has been shown to be stimulate activity and to efficiently remove excess damaged proteins. 


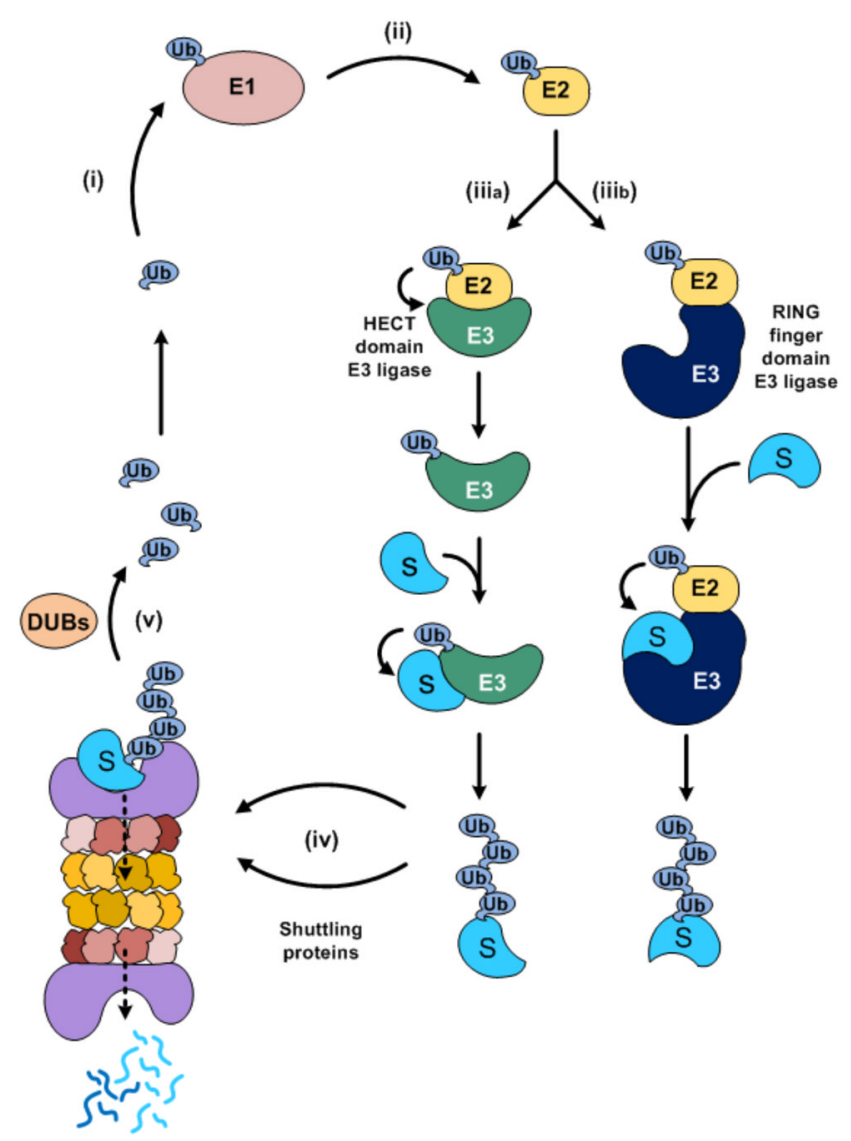

Figure 1. Schematic of the UPS

(i) Ubiquitin is activated by the ubiquitin-activating enzyme (E1), which uses ATP to form a high energy labile E1-thiol ester intermediate. (ii) The activated ubiquitin is transferred to a ubiquitin carrier protein (ubiquitin-conjugating enzyme; E2) to generate a similar thiol ester intermediate. (iiia) Activated ubiquitin can be transferred from the E2 to a HECT

(Homologous to the E6-AP C-Terminus) domain ubiquitin protein-ligase (E3) to generate a third labile thiol ester intermediate. It is then transferred to the substrate $(\mathrm{S})$, where, in most cases, a stable isopeptide bond between the activated carboxy terminal Gly76 of ubiquitin and an $\varepsilon \mathrm{NH}_{2}$ group of an internal lysine residue in the substrate is formed. Additional ubiquitin moieties are then added to generate a polyubiquitin chain. (iiib) Alternatively, activated ubiquitin can be transferred directly from E2 to an internal lysine residue in a substrate that is bound to a RING finger ubiquitin ligase. Polyubiquitin chains linked through Lys 48 of ubiquitin are characteristically associated with proteasomal degradation. However, linkages through several of the other seven lysines in ubiquitin have recently been shown to also target for proteasomal degradation ${ }^{119-122}$. In some cases, ubiquitin is conjugated to the $\mathrm{aNH}_{2}$ group of the target substrate (reviewed in REF. 24), and there are a few examples of conjugation to internal substrate Thr, Ser, or Cys residues ${ }^{122-128}$. Certain substrates are recognized by several ligases that act either under different conditions, sequentially or in parallel, recognizing different motifs in the substrate. Such a mechanism may be necessary to ascertain recognition and degradation of different domains in the substrate. (iv) Degradation of the ubiquitin-conjugated substrate to short peptides by the $26 \mathrm{~S}$ proteasome. The binding of ubiquitinated proteins to the proteasome can be direct or mediated or enhanced by shuttle proteins (v) Most of the ubiquitin chain is disassembled by deubiquitinating enzymes (DUBs), the rest is probably degraded along with the substrate. 
Select substrates are processed rather than completely degraded by the $26 \mathrm{~S}$ proteasome. This is due, at least in part, to unstructured sequences (for example, GlyAla repeats) that may inhibit entry into the proteasome, although additional mechanisms may be involved. 

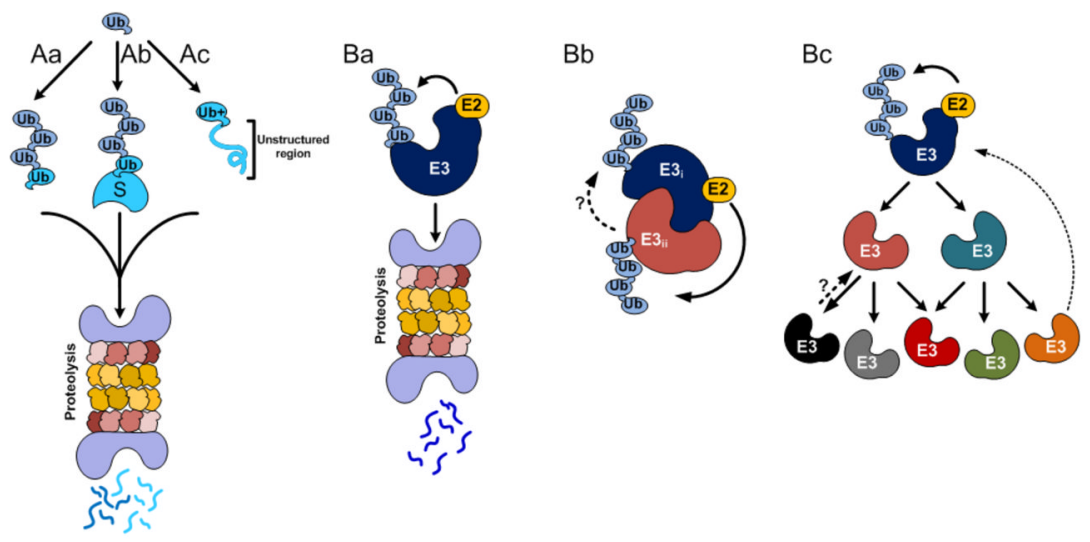

Figure 2. Modes of degradation of ubiquitin and ubiquitin-protein ligases

A. Ubiquitin can be degraded in three ways. (Aa) It can be degraded as a monomer following its polyubiquitylation (indicated in light blue). The process is probably slow, as the ubiquitin substrate molecule is short and lacks an unstructured tail that appears to be an important structural characteristic necessary for efficient proteasomal degradation. (Ab) Ubiquitin can be degraded with its conjugated substrate. The proximal part of the polyubiquitin chain is probably degraded along with the substrate, whereas the distal part is recycled by DUBs as free and reusable ubiquitin. (Ac) Ubiquitin with a C-terminal tail unstructured tail that is longer than 20 amino acid residues can be degraded efficiently by the proteasome. B. Modes of degradation of ubiquitin-protein ligases by ubiquitylation. (Ba) Degradation of a ligase mediated by self-ubiquitylation. Both RING and HECT domains-containing ligases can catalyze self-ubiquitylation, which generate polyubiquitin chains that target the proteins for proteasomal degradation. Self ubiquitylation is shown as occurring in cis (i.e. on the same molecule). However, as many E3s dimerize and may form higher order oligomers, ubiquitylation can also occur in trans as discussed in the text for Hrd1p. (Bb) Ubiquitylation of a ligase by a heterologous ligase. Whereas in the described cases of this mode of ubiquitylation the reaction is unidirectional (see Text and Figures 3 and 4), bidirectional ubiquitylation is also a possibility. (Bc) Hierarchical ubiquitylation of ligases. In this hypothetical scheme, one ligase targets several substrate ligases in a hierarchical manner. As has been described for several substrates of the ubiquitin system, one ligase substrate can be targeted by more than a single ligase (middle ligase in lower row and left ligase in middle row). The ligase at the top is targeted by self-ubiquitylation or by one of the ligases that it directly or indirectly regulates. 
A.

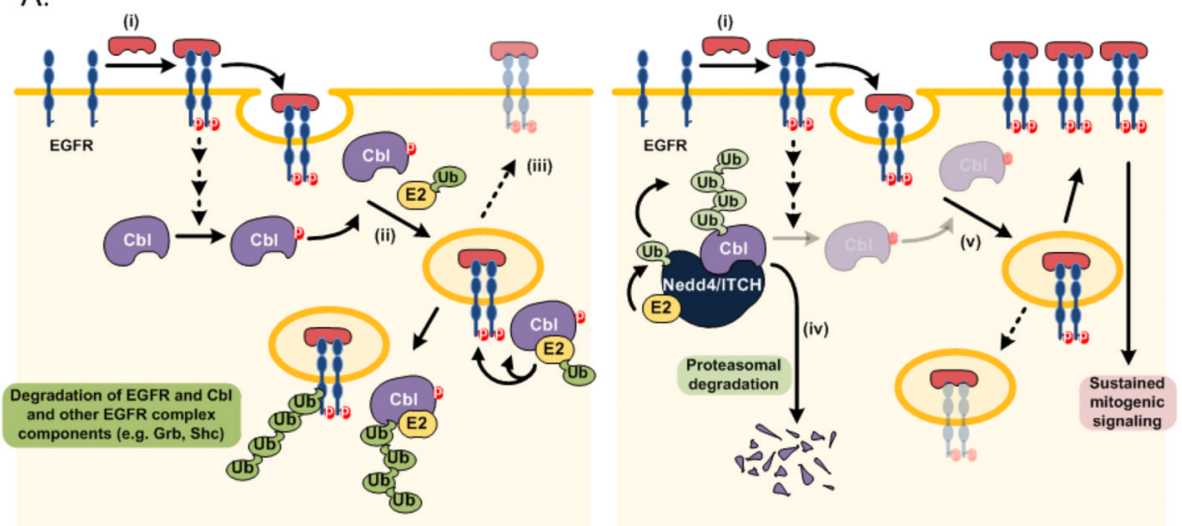

B.

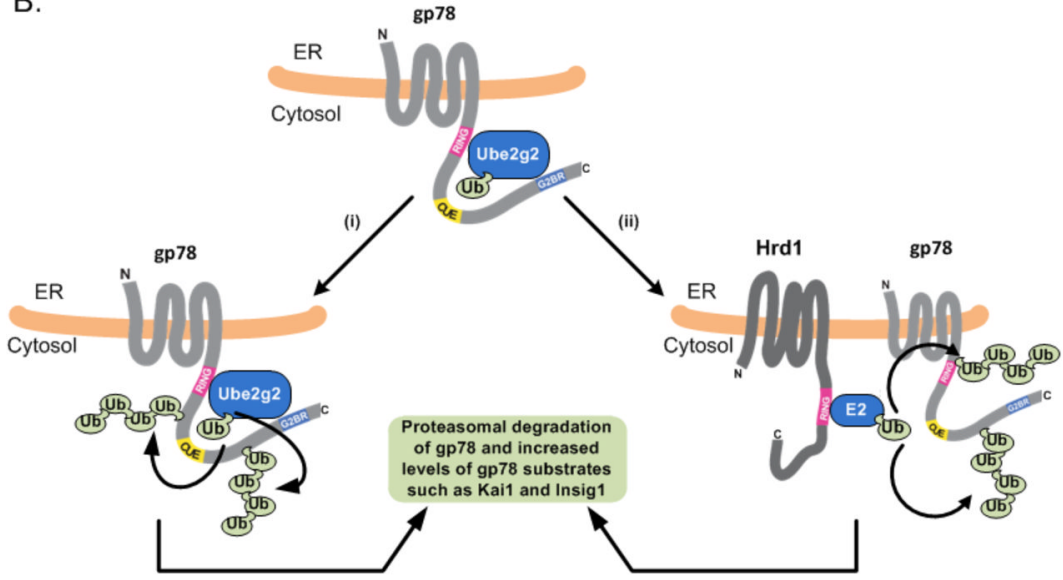

Figure 3. Targeting of Specific Ligases for Degradation by both Self- and Heterologous Ubiquitylation

A. Regulation of Cbl proteins and effects on signaling. Left hand side: (i) Binding of ligand induces dimerization and cross-phosphorylation of receptor tyrosine kinases (for example, EFGR - represented here) or receptor-associated tyrosine kinases as well as phosphorylation of other substrates, including other components of the signaling complex and $\mathrm{Cbl}$ proteins. The receptors together with other components of the signaling complex (not shown) are endocytosed and phosphorylated; Cbl proteins are recruited. (ii) Cbls mediate the ubiquitylation of receptors and associated proteins as well as self-ubiquitylation, resulting in targeting of receptors for lysosomal degradation and the destruction of $\mathrm{Cbl}$ molecules - whether this degradation is lysosomal or proteasomal has not been determined with certainty. (iii) The net result is decreased recycling of receptors to the cell surface and downregulation of signaling. Right hand side: (iv) Nedd4 family members target $\mathrm{Cbl}$ proteins for proteasomal degradation lowering the cellular levels of these E3s. (v) As a result ubiquitylation of activated receptor tyrosine kinase complexes is decreased. This results in attenuation of receptor down-regulation leading to increased mitogenic signaling. B. gp78 and ERAD. (i) gp78 is a polytopic RING finger E3 found in the ER that mediates selfubiquitylation using the E2, Ube2g2. This activity requires an intact gp78 RING finger, the ubiquitin binding CUE domain (whose function in this process is not yet clear) and a highly specific binding site for Ube2g2 that is distinct from the RING finger ${ }^{65}$. (ii) gp78 can additionally be targeted for ubiquitylation by a heterologous RING finger E3, Hrd1 (human Hrd1)/Synoviolin. This targeting is dependent on the Hrd1/Synoviolin RING finger but is independent of the gp78 RING finger. Both of these modes of degradation lead to proteasomal degradation of gp78 and consequently increased levels of gp78 substrates 
including Insig-1, and KAI1 (also known as CD82), which are regulators of cholesterol metabolism and a metastasis suppressor, respectively. 


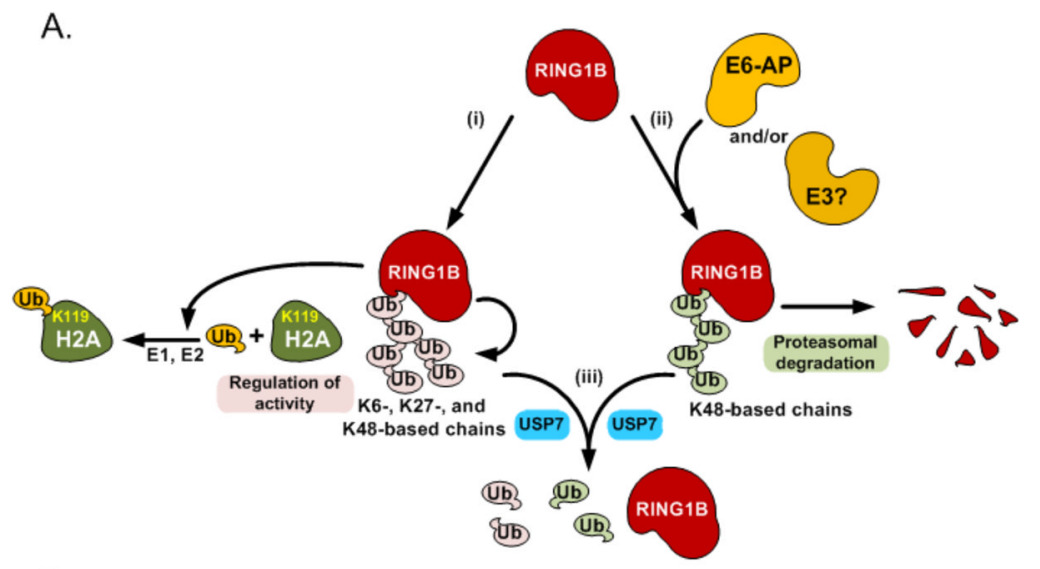

B.

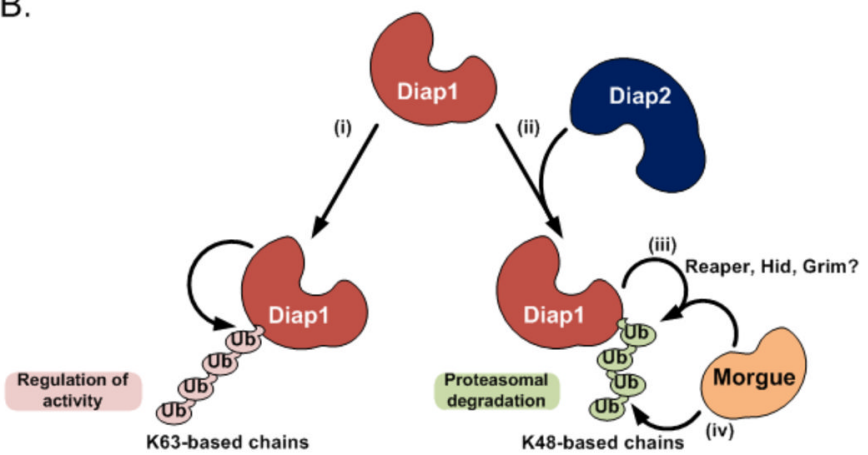

C.

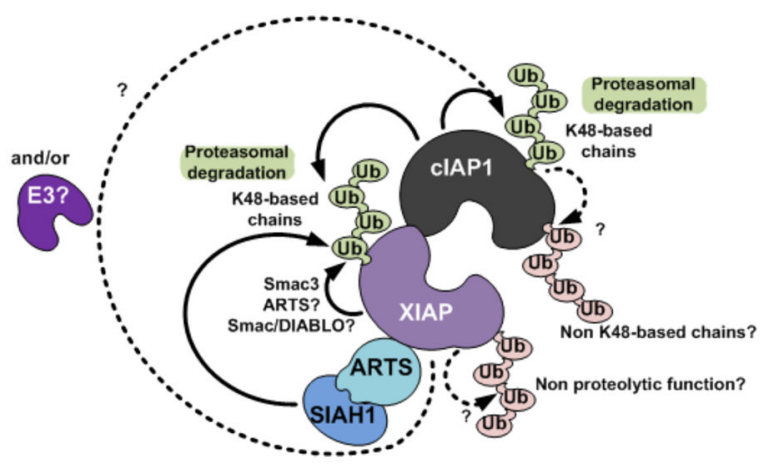

Figure 4. Regulation of specific ligases

A. Regulation of RING1B by ubiquitylation. (i) Self-ubiquitylation of RING1B generates 'non-canonical' - mixed (K6-, K-27-, and K-48-based) and multiply branched polyubiquitin chains that stimulate the monoubiquitinating activity of the ligase towards histone $\mathrm{H} 2 \mathrm{~A}$, but do not target it for degradation. (ii) The HECT E3 E6-AP (and possibly an additional ligase) targets RING1B for proteasomal degradation by generating K48-based polyubiquitin chains. Both the self and the E6-AP-catalyzed ubiquitylations target the same lysine residues on RING1B, thus preventing opposing and wasteful activities - destruction of an active and needed enzyme, or untoward activation of an un-needed enzyme. (iii) USP7 de-ubiquitylates both the activating and the proteasome-targeting polyubiquitin chains. Its activity releases free RING1B, thus enabling inactivation, and at the same time stabilization of RING1B without destroying it. B. Regulation of Diap1. (i) Self-ubiquitylation of Diap1 generates K63-based polyubiquitin chains that attenuate the ligase activity towards its substrates such as caspases. (ii) Diap2 targets Diap1 for proteasomal degradation by generating K48-based polyubiquitin chains on Diap1. (iii) Following an apoptotic stimulus, Reaper, Hid and 
possibly Grim (which are known as the RHG family) induce accelerated self-ubiquitylation of Diap1, generating K48-based chains that target the protein for degradation. (iv) Morgue generates K48-based chains either independently, or in conjunction with the RHG family members. These chains target Diap1 for proteasomal degradation. C. Regulation of mammalian IAPs. In mammals both cellular IAP1 (cIAP1) and X chromosome-linked IAP (XIAP) can catalyze their self-ubiquitylation, generating K48-based chains that probably target them for proteasomal degradation. It is not known whether, like D. melanogaster IAP1 (Diap1), they can also generate non K48-based chains that serve non-proteolytic functions. Intact cIAP1 (or its RING finger domain alone) was shown to catalyze formation of K48-based chains on XIAP1 that target it for degradation. It is not known whether XIAP and/or another ligase can catalyze a similar reaction, targeting cIAP1 for degradation. The ligase SIAH1 can mediate generation of K48-based chains that target XIAP for degradation. This reaction is mediated by ARTS that probably allows for association of SIAH1 and XIAP. Similar to the RHG family members, Smac3 (and possibly Smac/DIABLO and ARTS) can induce accelerated auto-ubiquitylation of XIAP, which leads to its degradation. Solid arrows mark experimentally established ubiquitylations, broken arrows denote putative ubiquitylations. Ubiquitin-conjugating enzymes (E2s) are not represented graphically in this figure to reduce complexity, but should be assumed to be involved in all ubiquitylation reactions. 
A.

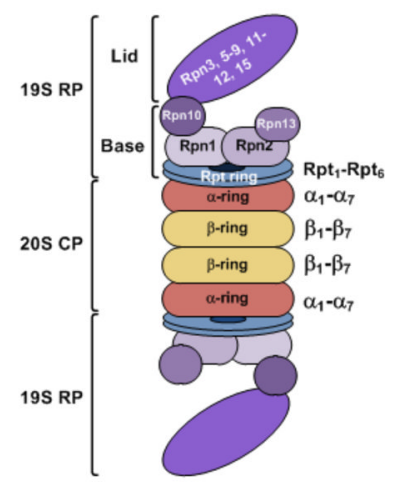

B.

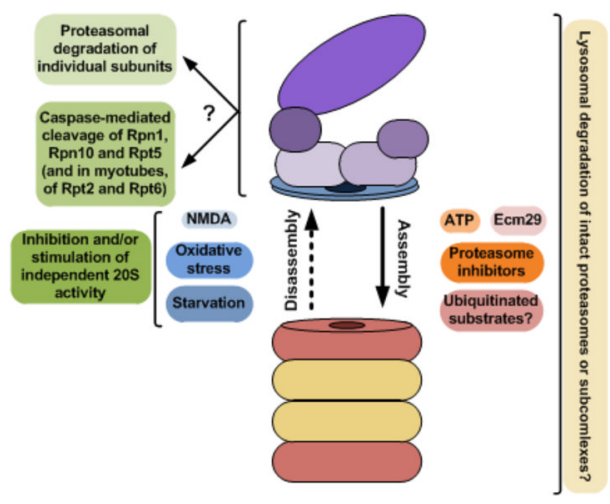

Figure 5. The $26 \mathrm{~S}$ proteasome and its regulation by degradation

A. Structure of the $26 \mathrm{~S}$ proteasome. The $26 \mathrm{~S}$ proteasome is comprised of two subcomplexes - the $20 \mathrm{~S}$ core catalytic particle (CP), and the $19 \mathrm{~S}$ regulatory particle (RP). The substrate is first bound, probably via its polyubiquitin chain(s), to specific subunits in the 19S RP. It is then probably unfolded by ATPases residing in the base of the RP (Rpt ring), and inserted via an open gate in the a-ring of the 20S CP into the proteolytic chamber. The ATPases are probably also involved in opening the interlacing chains that close the gate into the a-ring of the $\mathrm{CP}$, thus allowing entry of the unfolded substrate. The RP contains also $\operatorname{DUB}(\mathrm{s})$ that recycle ubiquitin and/or edit the polyubiquitin chain to enhance substrate binding to the RP and to recycle ubiquitin. A RP-associated ubiquitin ligase(s) may also function to adapt the chain length/type for optimal recognition by the RP. Proteolysis of the substrate is mediated by three pairs of proteolytically active $\beta$ subunits inside the proteolytic chamber, which includes two adjacent $\beta$ rings. B. Regulation of assembly and disassembly of the $26 \mathrm{~S}$ proteasome, and degradation of its subunits. The entire proteasome or its subcomplexes are probably degraded by the lysosome via microautophagy. Different factors control assembly (ATP, Ecm29, ubiquitylated substrates, and proteasome inhibitors, for example) and disassembly of the proteasome to its RP and CP (for example, NMDA ( $N$ methyl-D-aspartate) and different stresses, such as oxidative stress and starvation). The RP can be further disassembled into its individual subunits, which are probably degraded by proteasomes following ubiquitylation. For further reading on the proteasome structure and function, see REF. 88. 
Table 1

Regulatory modification of the UPS by ubiquitin and UBL

\begin{tabular}{|c|c|c|c|}
\hline Substrate & Targeting Enzyme(s) & Comments (substrate) & Refs. \\
\hline \multicolumn{4}{|c|}{ E3s as ubiquitylation targets of E3s } \\
\hline $\operatorname{Mdm} 2$ & Self & Primary p53 E3 & See text \\
\hline E6-AP & Self & $\begin{array}{l}\text { Targets p53 with HPV E6; notably self- ubiquitylation blocked in } \\
\text { vitro by p53 and HPV E6 suggesting substrate-mediated } \\
\text { inhibition of E6- AP }\end{array}$ & 129,130 \\
\hline Cbl E3s & $\begin{array}{l}\text { self, Nedd4 and Itch WW } \\
\text { domain HECT E3s }\end{array}$ & Critical E3s in modulating transmembrane signaling & See text \\
\hline gp78 & self, Hrd1/synoviolin & $\begin{array}{l}\text { Pro-metastatic ERAD E3, multiple substrates including KAI1, } \\
\text { INSIG-1 }\end{array}$ & See text \\
\hline TEB4 & Self & $\begin{array}{l}\text { ERAD E3; several substrates including the bile salt export pump } \\
\text { and iodothyronine deiodinase; mammalian ortholog of Doa10 }\end{array}$ & $131-133$ \\
\hline Hrd1p/Der3p & Self & $\begin{array}{l}\text { A major ERAD E3 of yeast implicated in degradation of multiple } \\
\text { substrates including HMG-CoA Reductase, the limiting enzyme } \\
\text { in sterol biosynthesis. }\end{array}$ & See text \\
\hline $\mathrm{Cdc} 20$ & $\mathrm{APC} / \mathrm{C}^{\mathrm{Cdh} 1}$ & $\begin{array}{l}\text { Substrate recognition element of APC/C; must be inactivated } \\
\text { after M phase }\end{array}$ & See Box1 \\
\hline Cdh1 & $\mathrm{APC} / \mathrm{C}^{\mathrm{Cdh} 1}, \mathrm{SCF}^{\mathrm{x}}$ & $\begin{array}{l}\text { Substrate recognition element of } \mathrm{APC} / \mathrm{C} \text {; must be inactivated in } \\
\text { G1 to inactivate APC/C }\end{array}$ & See Box 1 \\
\hline Skp2 & $\mathrm{APC}^{\mathrm{Cdh} 1}$ & $\begin{array}{l}\text { Substrate recognition element of SCF; F-box protein that targets } \\
\text { p } 27 \text { for degradation; Skp2 degradation prevents premature entry } \\
\text { into S phase }\end{array}$ & See Box 1 \\
\hline $\begin{array}{l}\text { F-box components of } \\
\text { SCF E3s: Cdc4, Grr1 }\end{array}$ & SCF, possibly other E3s. & $\begin{array}{l}\text { Degradation regulates free levels of these F-box proteins, } \\
\text { perhaps optimizing SCF function }\end{array}$ & 134 \\
\hline RING 1B & Self, E6-AP & PRC1 component, targets H2A for mono- ubiquitylation & See Text \\
\hline cIAP-1 & Self & $\begin{array}{l}\text { Targets itself for proteasomal degradation in RING-dependent } \\
\text { manner }\end{array}$ & See text \\
\hline DIAP-1 & Self, DIAP2 & D. melanogaster inhibitor of apoptosis & See text \\
\hline TRAF-2 & cIAP-1 & $\begin{array}{l}\text { Integral to NF- } \kappa \text { B signaling pathways and functions by } \\
\text { catalyzing non-proteasome-targeting K63 chains; cIAP-1 } \\
\text { together with TNFR-II targets TRAF- } 2 \text { for proteasomal } \\
\text { degradation }\end{array}$ & 135 \\
\hline XIAP & SIAH & $\begin{array}{l}\text { The mitochondrial protein ARTS (Apoptosis- Related protein in } \\
\text { the TGF- } \beta \text { Signaling pathway), which is a potential tumor } \\
\text { suppressor, serves as an obligatory adaptor in this process and is } \\
\text { lost in malignancies }\end{array}$ & See Text \\
\hline $\begin{array}{l}\text { BRCA1 (Breast } \\
\text { cancer- Associated 1) }\end{array}$ & HERC2 & $\begin{array}{l}\text { BRCA1 mutations or loss implicated in breast and ovarian } \\
\text { cancer; degraded in the absence of its binding partner, BARD1; } \\
\text { HECT E3 HERC suggested to be responsible for targeting of } \\
\text { BRCA1 for degradation in the absence of its binding partner } \\
\text { BARD1 }\end{array}$ & 136 \\
\hline FANCM & $\mathrm{SCF}^{\beta-\operatorname{TrCP}}$ & $\begin{array}{l}\text { Targeted for degradation before } M \text { phase, this prevents } \\
\text { inappropriate activation of FANC complex and chromosomal } \\
\text { abnormalities }\end{array}$ & 137 \\
\hline RNF8, RNF168 & HSV-ICP0 & $\begin{array}{l}\text { Targeting of these DNA repair E3s facilitates lytic activity of } \\
\text { Herpes Simplex Virus }\end{array}$ & 138 \\
\hline MARCH7 & self & $\begin{array}{l}\text { Implicated in neuronal development and lymphocyte } \\
\text { proliferation; deubiqutinated and stabilized by USP9X in the } \\
\text { cytosol and by USP7 in the nucleus }\end{array}$ & 139 \\
\hline Ro52 & self & $\begin{array}{l}\text { Normal function unknown; substrates include phosphorylated } \\
\text { IKK } \beta \text { when HTLV-1 Tax is expressed, resulting in } \\
\text { monoubiquitylation and downregulation of NF } \beta \text { B signaling; }\end{array}$ & 140,141 \\
\hline
\end{tabular}




\begin{tabular}{|c|c|c|c|}
\hline Substrate & Targeting Enzyme(s) & Comments (substrate) & Refs. \\
\hline & & $\begin{array}{l}\text { target of autoantibodies in Sjogren's syndrome; deubiquitylated } \\
\text { by Usp4 (see DUB table below) }\end{array}$ & \\
\hline \multicolumn{4}{|c|}{ DUBs as ubiquitylation substrates } \\
\hline Usp6 & Unknown & $\begin{array}{l}\text { Oncogenic DUB; monoubiquitylation is dependent on } \\
\text { calmodulin binding; function unknown }\end{array}$ & 142 \\
\hline UCH-L1 & Unknown & $\begin{array}{l}\text { Parkinson's disease-associated DUB; monoubiquitylation serves } \\
\text { to inhibit interaction with ubiquitylated potential substrates }\end{array}$ & 143 \\
\hline Ataxin-3 & gp78, others? & $\begin{array}{l}\text { Implicated in protein quality control including ERAD; implicated } \\
\text { in poly-Q disease spino- cerellebar ataxia type } 3 \text { or Machado- } \\
\text { Joseph disease; ubiquitylation reported to increases cataytic } \\
\text { activity and not alter preference for K63 chains; ubiquitylation } \\
\text { by gp78 targets for degradation }\end{array}$ & $144,145,146$ \\
\hline Usp4 (UnpEL) & Ro52 (see above) & Function unknown & 147 \\
\hline Usp7 (HAUSP) & $\begin{array}{l}\text { HSV-ICP0; endogenous } \\
\text { E3s not established }\end{array}$ & $\begin{array}{l}\text { Targeted for ubiquitylation and proteasomal degradation by } \\
\text { ICP0; also reported to be both ubiquitylated and Neddylated in } \\
\text { cells not expressing ICP0 }\end{array}$ & 148,149 \\
\hline Usp25 & Unknown & $\begin{array}{l}\text { Contains UBA and UIM important for monoubiquitylation on } \\
\text { specific Lys and for function; monoubiquitylation activates DUB } \\
\text { activity towards its substrate, MyBPC1; sumoylation of the same } \\
\text { Lys has opposing effect }\end{array}$ & 150 \\
\hline \multicolumn{4}{|c|}{ E2s as self-ubiquitylation or E3 targets } \\
\hline Ubc7p & Self & $\begin{array}{l}\text { Yeast ERAD E2; functions with Hrd1p and Doa10p; } \\
\text { ubiquitylation dependent on intrinsic activity and does not } \\
\text { require other E2s; self- ubiquitylation occurs in vitro, whether an } \\
\text { E3 is required for ubiquitylation in vivo is unknown }\end{array}$ & $31,151,152$ \\
\hline Ubc6p & Doa10p & $\begin{array}{l}\text { Yeast ERAD E2; ubiquitylation dependent on both Doa10p and } \\
\text { itself }\end{array}$ & 153 \\
\hline UbcH10 & $\mathrm{APC}^{\mathrm{Cdh} 1}$ & $\begin{array}{l}\text { Critical E2 in APC/C function; degraded by the E3 as part of the } \\
\text { process leading to APC/C inactivation in G1 }\end{array}$ & See Box 1 \\
\hline \multicolumn{4}{|c|}{ Cross-targeting between ubiquitination enzymes and UBL enzymes } \\
\hline Ubc9p & Self & $\begin{array}{l}\text { Yeast E2 that mediates SUMO modification; self-sumoylation } \\
\text { decreases activity towards some substrates }\end{array}$ & 154 \\
\hline E2-25K (Ube2K) & Ubc9 & $\begin{array}{l}\text { Sumoylation on N-terminal helix decreases ability to be loaded } \\
\text { by ubiquitin E1 }\end{array}$ & 155 \\
\hline CRL Cullins & $\begin{array}{l}\text { Self (Rbx1 component of } \\
\text { CRLs) and DCN4 }\end{array}$ & Modification with Nedd8 critical to activation of CRLs & $\begin{array}{l}\text { reviewed in Ref } \\
156\end{array}$ \\
\hline
\end{tabular}

